\title{
Investigating the anatomy of magnetosheath jets - MMS observations
}

\author{
Tomas Karlsson $^{1}$, Ferdinand Plaschke ${ }^{2}$, Heli Hietala ${ }^{3}$, Martin Archer $^{4}$, Xóchitl Blanco-Cano ${ }^{5}$, Primož Kajdič ${ }^{5}$, \\ Per-Arne Lindqvist ${ }^{1}$, Göran Marklund ${ }^{1}$, and Daniel J. Gershman ${ }^{6}$ \\ ${ }^{1}$ Space and Plasma Physics, School of Electrical Engineering, KTH Royal Institute of Technology, \\ 10044 Stockholm, Sweden \\ ${ }^{2}$ Space Research Institute, Austrian Academy of Sciences, Schmiedlstrasse 6, 8042 Graz, Austria \\ ${ }^{3}$ Department of Earth, Planetary, and Space Sciences, University of California Los Angeles, 603 Charles E. Young Drive East, \\ Slichter Hall 6844D, Los Angeles, CA 90095-1567, USA \\ ${ }^{4}$ School of Physics \& Astronomy, Queen Mary University of London, London, E1 4NS, UK \\ ${ }^{5}$ Instituto de Geofísica Universidad Nacional Autónoma de México, Ciudad Universitaria, CDMX, México \\ ${ }^{6}$ NASA Goddard Space Flight Center, Greenbelt, Maryland, USA
}

Correspondence: Tomas Karlsson (tomas.karlsson@ee.kth.se)

Received: 12 October 2017 - Revised: 29 January 2018 - Accepted: 3 April 2018 - Published: 23 April 2018

\begin{abstract}
We use Magnetosphere Multiscale (MMS) mission data to investigate a small number of magnetosheath jets, which are localized and transient increases in dynamic pressure, typically due to a combined increase in plasma velocity and density. For two approximately hour-long intervals in November, 2015 we found six jets, which are of two distinct types. (a) Two of the jets are associated with the magnetic field discontinuities at the boundary between the quasi-parallel and quasi-perpendicular magnetosheath. Straddling the boundary, the leading part of these jets contains an ion population similar to the quasi-parallel magnetosheath, while the trailing part contains ion populations similar to the quasi-perpendicular magnetosheath. Both populations are, however, cooler than the surrounding ion populations. These two jets also have clear increases in plasma density and magnetic field strength, correlated with a velocity increase. (b) Three of the jets are found embedded within the quasi-parallel magnetosheath. They contain ion populations similar to the surrounding quasi-parallel magnetosheath, but with a lower temperature. Out of these three jets, two have a simple structure. For these two jets, the increases in density and magnetic field strength are correlated with the dynamic pressure increases. The other jet has a more complicated structure, and no clear correlations between density, magnetic field strength and dynamic pressure. This jet has likely interacted with the magnetosphere, and contains ions similar
\end{abstract}

to the jets inside the quasi-parallel magnetosheath, but shows signs of adiabatic heating. All jets are associated with emissions of whistler, lower hybrid, and broadband electrostatic waves, as well as approximately $10 \mathrm{~s}$ period electromagnetic waves with a compressional component. The latter have a Poynting flux of up to $40 \mu \mathrm{W} \mathrm{m} ~^{-2}$ and may be energetically important for the evolution of the jets, depending on the wave excitation mechanism. Only one of the jets is likely to have modified the surrounding magnetic field into a stretched configuration, as has recently been reported in other studies. None of the jets are associated with clear signatures of either magnetic or thermal pressure gradient forces acting on them. The different properties of the two types also point to different generation mechanisms, which are discussed here. Their different properties and origins suggest that the two types of jets need to be separated in future statistical and simulation studies.

Keywords. Magnetospheric physics (magnetosheath; plasma waves and instabilities; solar wind-magnetosphere interactions) 


\section{Introduction}

Small-scale, transient increases in magnetosheath dynamic pressure have recently attracted increased attention (e.g. Němeček et al., 1998; Savin et al., 2008; Hietala et al., 2009; Archer et al., 2012; Plaschke et al., 2013). They have gone under a number of different denominations, here we will call them "magnetosheath jets", or sometimes simply "jets".

Magnetosheath jets can be an important factor in the solar wind-magnetosphere interaction, since their increased momentum may cause the magnetosphere to deform locally when it is impacted by the jets (Shue et al., 2009; Amata et al., 2011). Such deformations can launch surface waves (Dmitriev and Suvorova, 2012; Archer et al., 2012) or compressional waves (Plaschke et al., 2009; Archer et al., 2013a, b), initiate ionospheric flow enhancements (Hietala et al., 2012), trigger local reconnection (Hietala et al., 2018), or lead to direct penetration of plasma into the magnetosphere (Gunell et al., 2012; Karlsson et al., 2012; Savin et al., 2012; Dmitriev and Suvorova, 2015). Furthermore, a type of dayside auroral form, the "throat" aurora, has recently been suggested to be a direct consequence of magnetosheath jets interacting with the magnetopause (Han et al., 2017). Plaschke et al. (2016) have recently estimated that jet impact on the magnetopause should be very common, with jets larger than $2 R_{\mathrm{E}}$ (Earth radii) impacting 3-9 times an hour, depending on the interplanetary magnetic field (IMF) direction.

Several different definitions of magnetosheath jets have been used in earlier studies. Either the dynamic pressure is required to be larger than some value related to the background magnetosheath, often defined by a running average (e.g Archer and Horbury, 2013; Karlsson et al., 2015b; Gunell et al., 2014; Gutynska et al., 2015), or to exceed some value related to the solar wind dynamic pressure (e.g. Amata et al., 2011; Hietala et al., 2012; Plaschke et al., 2013; Dmitriev and Suvorova, 2015). The increase in dynamic pressure of the magnetosheath jets is due to either an increase in flow velocity (Archer et al., 2012; Plaschke et al., 2013), in density (Savin et al., 2008; Archer and Horbury, 2013; Karlsson et al., 2012, 2015b), or in both (Amata et al., 2011; Archer and Horbury, 2013; Plaschke et al., 2013; Karlsson et al., 2015b).

The properties of magnetosheath jets have been studied both on a case study basis and statistically, and some of their properties have begun to be determined. Their scale sizes are of the order of $1 R_{\mathrm{E}}$ (e.g. Savin et al., 2008; Archer et al., 2012; Hietala et al., 2012), with comparable dimensions parallel and perpendicular to the propagation velocity (Plaschke et al., 2016). Some jets are reported to be associated with an increase in the magnetic field strength, but not all (e.g. Němeček et al., 1998; Savin et al., 2008; Hietala et al., 2009, 2012). In this context it should be mentioned that so-called paramagnetic plasmoids (which are always associated with an increased magnetic field strength) are localized density enhancements $(>50 \%)$, often associated with an increased velocity, and are likely to be closely related to magnetosheath jets (Karlsson et al., 2015b).

Magnetosheath jets and plasmoids may be associated with plasma wave emission, similar to what has been found for fast flows in the magnetotail (e.g. Chaston et al., 2012; Ergun et al., 2015; Stawarz et al., 2015). So far this has only been studied by Gunell et al. (2014), who report that jets are associated with increased amplitudes of lower hybrid and electron whistler waves.

In two large statistical investigations Archer and Horbury (2013) show that jets with a density increase larger than about $40 \%$ are also associated with a magnetic field increase, while Plaschke et al. (2013) report on a distribution of magnetic field changes. This distribution ranges from magnetic field decreases to increases, but with a maximal probability for a small increase. Magnetosheath jets are also generally reported to be associated with a lower ion temperature than that of the surrounding magnetosheath plasma, usually with a very low temperature anisotropy (Shue et al., 2009; Archer et al., 2012; Dmitriev and Suvorova, 2012; Archer and Horbury, 2013; Plaschke et al., 2013).

Magnetosheath jets are most commonly observed downstream of the quasi-parallel bow shock, and the upstream IMF is steady for a large majority of the jets (Archer and Horbury, 2013; Plaschke et al., 2013). However, a minority of jets are related to IMF discontinuities (Archer et al., 2012; Dmitriev and Suvorova, 2012).

Several theories for the formation of magnetosheath jets have been considered, but presently interest is focussed on two main mechanisms. Based on the observation that some jets are associated with IMF discontinuities, Archer et al. (2012) suggested that the interaction of solar wind discontinuities with the bow shock was responsible for the jet formation. This was further based on simulations by Lin et al. (1996b), who showed that discontinuity-bow shock interaction resulted in highly stretched magnetic field lines, which can accelerate the plasma after it has crossed the bow shock. The largest acceleration was found to occur for discontinuities associated with changes from a quasi-parallel to a quasiperpendicular bow shock, or vice versa. The limited scale sizes of the jets were speculated to result from some type of interaction between the discontinuity and either the quasiparallel bow shock or the foreshock plasma upstream of it (Archer et al., 2012).

The second main theory was suggested by Hietala et al. (2009) and Hietala and Plaschke (2013) and was based on the observation that the majority of jets occur behind the quasiparallel bow shock. They suggested that jets can form as the solar wind interacts with the rippled bow shock associated with the quasi-parallel configuration. When the solar wind encounters a locally inclined part of the shock, the RankineHugoniot jump conditions (e.g. Baumjohann and Treumann, 2012) allow it to cross the shock without much change in velocity. The plasma will still be compressed, and this results in a dynamic pressure downstream of the shock that can even be 
larger than the upstream value. The scale size of the jet is determined by the scale of the bow-shock rippling, which was suggested to be related to the phenomenon of a short largeamplitude magnetic field structure (SLAMS), which has dimensions of the order of $1 R_{\mathrm{E}}$ (Schwartz and Burgess, 1991).

While jets have been studied for close to 20 years, many of their detailed properties are not well established, and are based on relatively few case studies. We will here revisit some of these properties by using data from the state-ofthe-art instruments of the Magnetospheric Multiscale (MMS) mission. Recently Plaschke et al. (2017) studied the smallscale internal structure of a magnetosheath jet, using hightime-resolution MMS observations. We will instead investigate the large- and meso-scale properties of a small number of jets, what we may call the anatomy of the jets, with particular emphasis on how these properties relate to the main theories of formation described above.

We will present the data in Sects. 2 and 3. The interpretation and discussion will take place in Sect. 4. Section 5 contains a summary and conclusions.

\section{MMS data}

The four spacecraft of the MMS mission (Burch et al., 2016; Fuselier et al., 2016) were successfully launched on 12 March 2015 with an inclination of $28^{\circ}$, and science operations began in September 2015. During Science Phase 1 a (September 2015-February 2016) the apogee was $12 R_{\mathrm{E}}$, and an appreciable part of the orbit was spent in the magnetosheath, since the position of the magnetopause (which was the main region of interest) varies considerably with the solar wind pressure. The spacecraft separation close to apogee in November 2015, where the data from this study are from, typically varied between 10 and $20 \mathrm{~km}$, with the satellites in a tetrahedron configuration.

The MMS spacecraft are equipped with an extensive suite of state-of-the-art instruments (Baker et al., 2016; Torbert et al., 2016). From the electric-field double probe (EDP) instrument (Lindqvist et al., 2016; Ergun et al., 2016), we use fast survey mode electric field data, where the full electric field vector is sampled at $32 \mathrm{~s}^{-1}$, and on-board spectrograms sampled at $0.5 \mathrm{~s}^{-1}$. From the fluxgate magnetometer (FGM) instrument (Russell et al., 2016) we use Slow survey mode magnetic field data $\left(8 \mathrm{~s}^{-1}\right)$, from the search coil magnetometer (SCM) (Le Contel et al., 2016) the on-board magnetic field spectrograms $\left(0.5 \mathrm{~s}^{-1}\right)$, and from the fast plasma investigation (FPI) (Pollock et al., 2016) fast survey ion and electron moments, and ion distributions $\left(0.22 \mathrm{~s}^{-1}\right)$.

Due to the small separation of the MMS spacecraft compared to the phenomena studied here, we mainly use data from MMS1, except for the calculation of the current density and thermal pressure gradients, where multi-spacecraft methods are used. All vector quantities and positions are given in the geocentric solar ecliptic (GSE) coordinate system.

\section{Observations}

We will investigate two approximately hour-long intervals in November 2015, each containing three magnetosheath jets.

\subsection{Overview, 14 November 2015}

Figure 1a shows an overview of MMS1 observations on 14 November 2015 00:00-18:00 UT. After several full and partial magnetopause crossings, the spacecraft finally passed into the magnetosheath, close to the subsolar point, at around 03:17 UT. It then spent several hours in the magnetosheath (with a brief excursion into the magnetosphere at around 12:36 UT), until reentering the magnetosphere at 14:27 UT. Between approximately 07:40 and 11:00 UT several time periods with a widening of the ion energy distributions, typical for the magnetosheath behind the quasi-parallel bow shock (Fuselier et al., 1991; Chang et al., 2000) were observed. As a general tendency these regions have a larger level of fluctuations of the dynamic pressure, and in particular there are several instances of large, isolated increases in the dynamic pressure $P_{\text {dyn }}=m_{\mathrm{p}} n_{\mathrm{i}} v_{\mathrm{i}}^{2}$ between 09:40 and 10:10 UT (see panel a4). (Here $m_{\mathrm{p}}$ is the proton mass, $n_{\mathrm{i}}$ the ion number density, and $\boldsymbol{v}_{\mathrm{i}}$ the ion velocity.)

In Fig 1b we show MMS1 observations from the time interval 09:30-10:20 UT in more detail. We have also included the magnetic field and associated cone angle (defined as the angle between the direction of IMF and the SunEarth line) observed by THEMIS B (panels b6-b7), which was situated in the solar wind at $\boldsymbol{r}_{\mathrm{GSE}}=(53,30,5) R_{\mathrm{E}}$. Using the THEMIS solar wind velocity in the GSE $x$ direction of $425 \mathrm{~km} \mathrm{~s}^{-1}$ yields a travel time of around $10 \mathrm{~min}$ to the bow shock nose. To this should be added a $2-4 \mathrm{~min}$ for propagation through the magnetosheath to MMS1. There are large uncertainties to this estimate, due to the offset of THEMIS B from the Sun-Earth line, and due to the varying directions of normals to solar wind discontinuities (Mailyan et al., 2008). We have instead manually shifted the THEMIS data by $21 \mathrm{~min}$. This results in a good correlation with the MMS1 data. In particular the discontinuities observed by THEMIS B at 09:36:50, 09:50:40, and 10:01-10:05 UT line up well with similar structures in the MMS1 magnetic field data. Furthermore, between 09:36:50 and 10:01 UT the generally smaller IMF cone angle coincides well with a region of suprathermal broadening of the ion energies (panel b8). We will refer to such regions of the magnetosheath as "the quasi-parallel magnetosheath", and all other regions as "the quasi-perpendicular magnetosheath" (Fuselier, 1994). The depression of the quasi-parallel magnetosheath magnetic field strength here may indicate that this region is an example of a so-called magnetosheath cavity (Katırcioglu et al., 2009). Note that since the MMS observations take place so close to the Sun-Earth line, we can take the cone angle as a proxy for the angle between the bow shock normal upstream of the spacecraft and the IMF. 

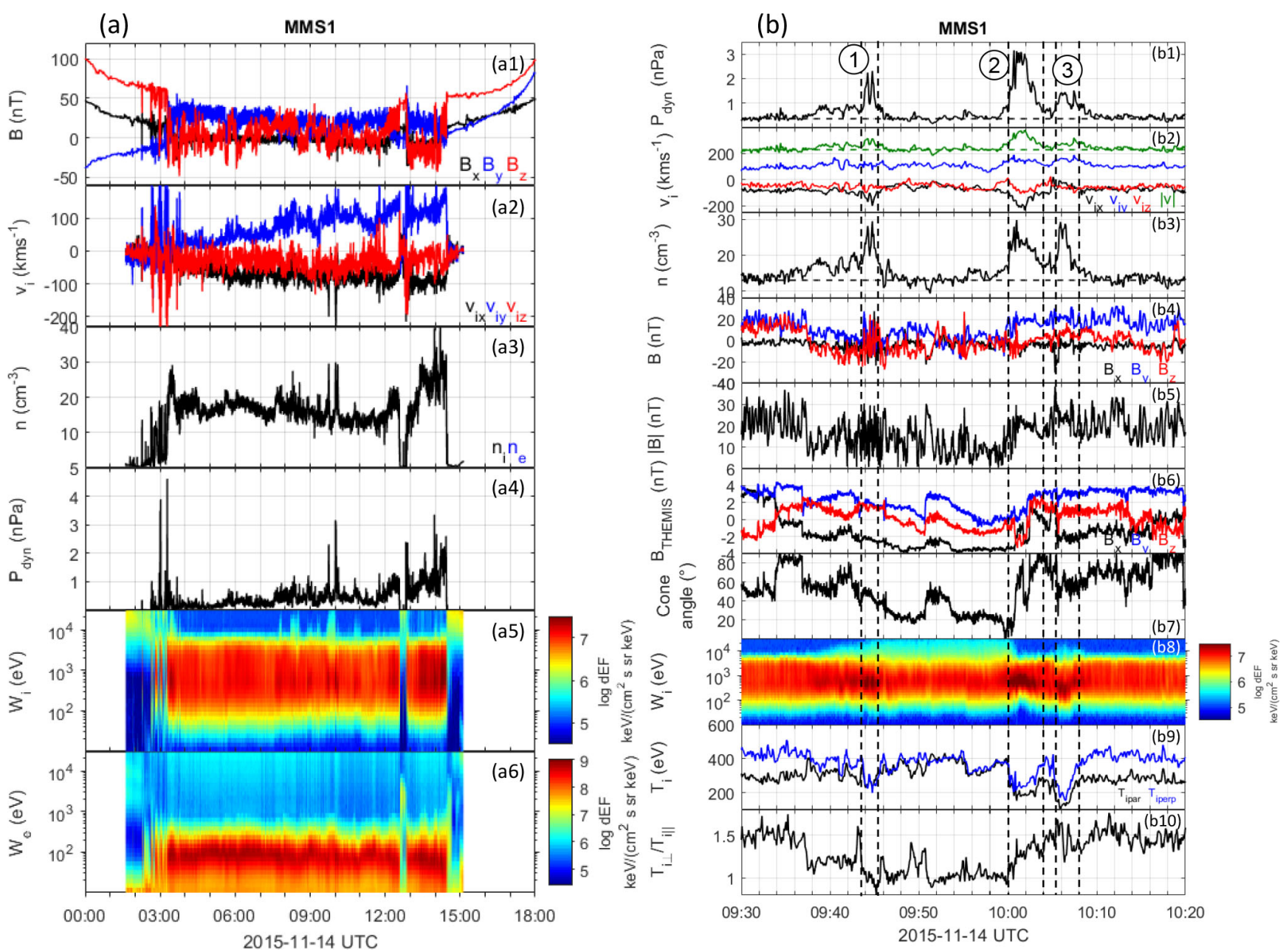

Figure 1. (a) Overview of MMS1 measurements in the magnetosheath on 14 November 2015. From top to bottom is shown magnetic field $\boldsymbol{B}$, ion velocity $\boldsymbol{v}_{\mathrm{i}}$, ion and electron density $\left(n_{\mathrm{i}}\right.$ and $\left.n_{\mathrm{e}}\right)$, dynamic pressure $P_{\mathrm{dyn}}$, and omnidirectional ion and electron differential energy flux. (b) From top to bottom: $P_{\text {dyn }}$ (with three localized increases indicated), $\boldsymbol{v}_{\mathrm{i}}$ and $\left|\boldsymbol{v}_{\mathrm{i}}\right|, n_{\mathrm{i}}, \boldsymbol{B},|\boldsymbol{B}|$, THEMIS B magnetic field (shifted by 21 min), THEMIS B magnetic field cone angle, omnidirectional ion differential energy flux, ion and parallel and perpendicular ion temperatures $\left(T_{i} \perp\right.$, $\left.T_{\mathrm{i} \|}\right)$, and ion temperature anisotropy $\left(T_{\mathrm{i} \perp} / T_{\mathrm{i} \|}\right)$.

During the time interval shown in Fig. 1b, there are three distinct increases in the dynamic pressure (marked 1-3), which were observed by MMS1 at a position of $r_{\mathrm{GSE}}=(11.8,0.9,-0.8) R_{\mathrm{E}}$. In panel (b1) a manually determined background level of $P_{\mathrm{dyn}}=0.36 \mathrm{nPa}$ is indicated. All three enhancements in $P_{\text {dyn }}$ fulfill the magnetosheath jet threshold criterion of twice the background dynamic pressure of Archer and Horbury (2013). We have indicated the extent of the jets with vertical lines, where the jets are defined as regions where this criterion is fulfilled, neglecting small excursions below the threshold. Structures 1 and 2 also fulfill the criterion of Archer et al. (2012) of a minimum increase in $P_{\text {dyn }}$ of $1.5 \mathrm{nPa}$. We will from now on call these structures "(magnetosheath) jets no. 1-3". Below we will investigate their properties in detail.

\subsubsection{Velocity and plasma properties}

Jets no. 1 and 2 have a clear increase in the $x$ component of the plasma flow velocity, resulting in a velocity more oriented along the Sun-Earth line than the background magnetosheath flow. This is a typical property of jets in the subsolar magnetosheath (Gunell et al., 2012; Karlsson et al., 2012; Hietala et al., 2012; Archer and Horbury, 2013; Hietala and Plaschke, 2013; Plaschke et al., 2013). In Fig. 2 we have used the velocities of these two jets (determined as an average over a small region around the maximum dynamic pressure of the structure) to back-trace the position at the bow shock from which we assume that the jets have emanated. (Here we assume that the flow velocity is constant during their motion across the magnetosheath.) As expected, the jets are seen to originate from close to the subsolar bow shock. However, jet no. 3 has a much smaller $x$ component of the velocity, and 


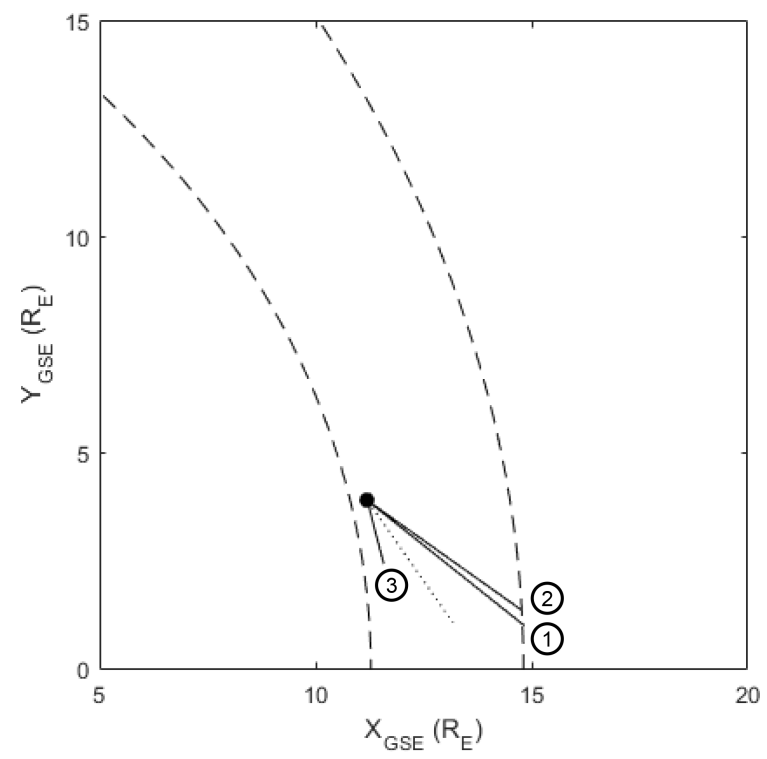

Figure 2. Back-tracking of jets no. 1 and 2 from their point of observations in the GSE $x-y$ plane (filled black circle) to the model bow shock, assuming a constant propagation velocity. For jet no. 3 , we back-track for an arbitrary time interval of $1 \mathrm{~min}$. The dotted line shows the direction of the background magnetosheath flow. Also shown are the model magnetopause and bow shock locations (Chao et al., 2002).

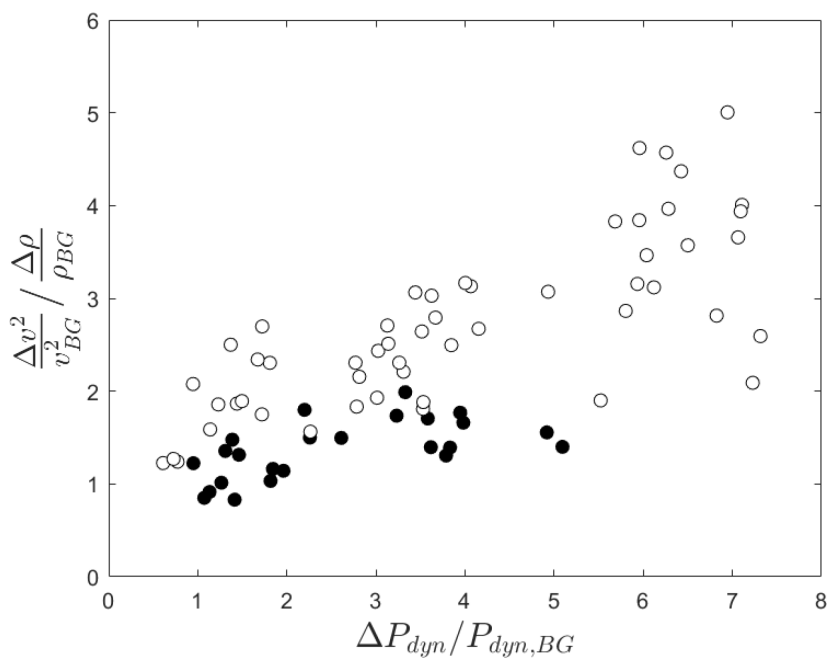

Figure 3. Relative contribution of the density changes $\Delta \rho_{\text {rel }}=$ $\Delta \rho / \rho_{\mathrm{BG}}$ and changes in the squared velocity $\Delta v_{\mathrm{i}, \text { rel }}^{2}=\Delta \boldsymbol{v}_{\mathrm{i}}^{2} / v_{\mathrm{i}, \mathrm{BG}}^{2}$ versus change in dynamic pressure (all quantities normalized to their background value). Black (white) data points are from jet no. 1 (jet no. 2), from the interval indicated in Fig. 1.

is moving almost exclusively in the $y$ direction. We find it probable that this jet has interacted with the magnetopause, similar to what has been reported by Shue et al. (2009) and Amata et al. (2011). An alternative interpretation is that the flows associated with jet no. 3 are due to a stirring of the background magnetosheath plasma by jet no. 2, as reported by Plaschke et al. (2017). This is, however, unlikely since the temperature of jet no. 3 is much lower than the surrounding magnetosheath plasma, and the density is higher. These are signatures of a jet, rather than of a stirred background plasma.

All three jets are associated with density enhancements of over $50 \%$, and therefore also fulfill the plasmoid criterion used by Karlsson et al. (2012, 2015b). Jets no. 1 and 2 would be classified as "fast plasmoids" in the terminology of those studies. Regarding the relative contribution of density and velocity increases to the dynamic pressure increase, jets no. 1 and 2 show somewhat different behaviours. In Fig. 3 is plotted the relative contribution of the density changes from the background value $\Delta \rho_{\text {rel }}=\Delta \rho / \rho_{\mathrm{BG}}$ and changes in the squared velocity $\Delta v_{\mathrm{i}, \text { rel }}^{2}=\Delta \boldsymbol{v}_{\mathrm{i}}^{2} / v_{\mathrm{i}, \mathrm{BG}}^{2}\left(\right.$ where $\rho=m_{\mathrm{p}} n_{\mathrm{i}}$, and both quantities are normalized to their respective background values), as a function of the relative change in dynamic pressure $\Delta P_{\text {dyn, rel }}=\Delta P_{\text {dyn }} / P_{\text {dyn, BG }}$. Archer and Horbury (2013) have shown that there is a continuous distribution in the relative importance of the two factors, taken over a large sample of jet data points. Jet no. 1 shows a relatively constant ratio of $\Delta v_{\mathrm{i}, \text { rel }}^{2}$ to $\Delta \rho_{\text {rel }}$ over the whole event. For jet no. 2 there is larger spread, with the dynamic pressure change being generally more velocity-driven, and with a systematically larger velocity contribution for the higher dynamic pressures within the jet.

Figure 4 shows ion distribution functions in the planes formed by the direction of the local values of magnetic field $\boldsymbol{B}, \boldsymbol{E} \times \boldsymbol{B}$, and $\boldsymbol{E}_{\perp}$ (where $\boldsymbol{E}$ is the electric field and $\perp$ refers to the projection perpendicular to $\boldsymbol{B}$ ). Shown are distribution function from within jets no. 1-3, and for reference distribution functions from quasi-parallel and quasi-perpendicular magnetosheath regions without any jets.

For jet no. 1 the ion distribution is very similar to the distribution for the general quasi-parallel magnetosheath, in that it is quite isotropic (although with a lower temperature, as can be verified by the temperatures in Fig. 1). The whole distribution has a displacement from the origin, which indicates a fluid velocity of the jet at an angle to the magnetic field of around $45^{\circ}$.

For jet no. 2, the situation is more complicated. Being collocated with the magnetic field discontinuity marking the transition from a quasi-parallel to a quasi-perpendicular magnetosheath configuration, it contains two types of ion populations. At the leading edge of the jet, a distribution function from approximately 10:00:49 UTC is shown in Fig. 4b. The distribution is similar to those of panels (a) and (e) in that it is relatively isotropic. At a later time (approx. 10:01:38 UTC) a distribution closer to the trailing part of jet no. 2 is shown in panel (c). The ion distribution is here more anisotropic, and similar to the distribution from the quasi-perpendicular magnetosheath, as shown in panel (f), with an added average or fluid velocity with a similar direction and size as that of panel (b). Figure 1 also shows that the plasma inside the jet 

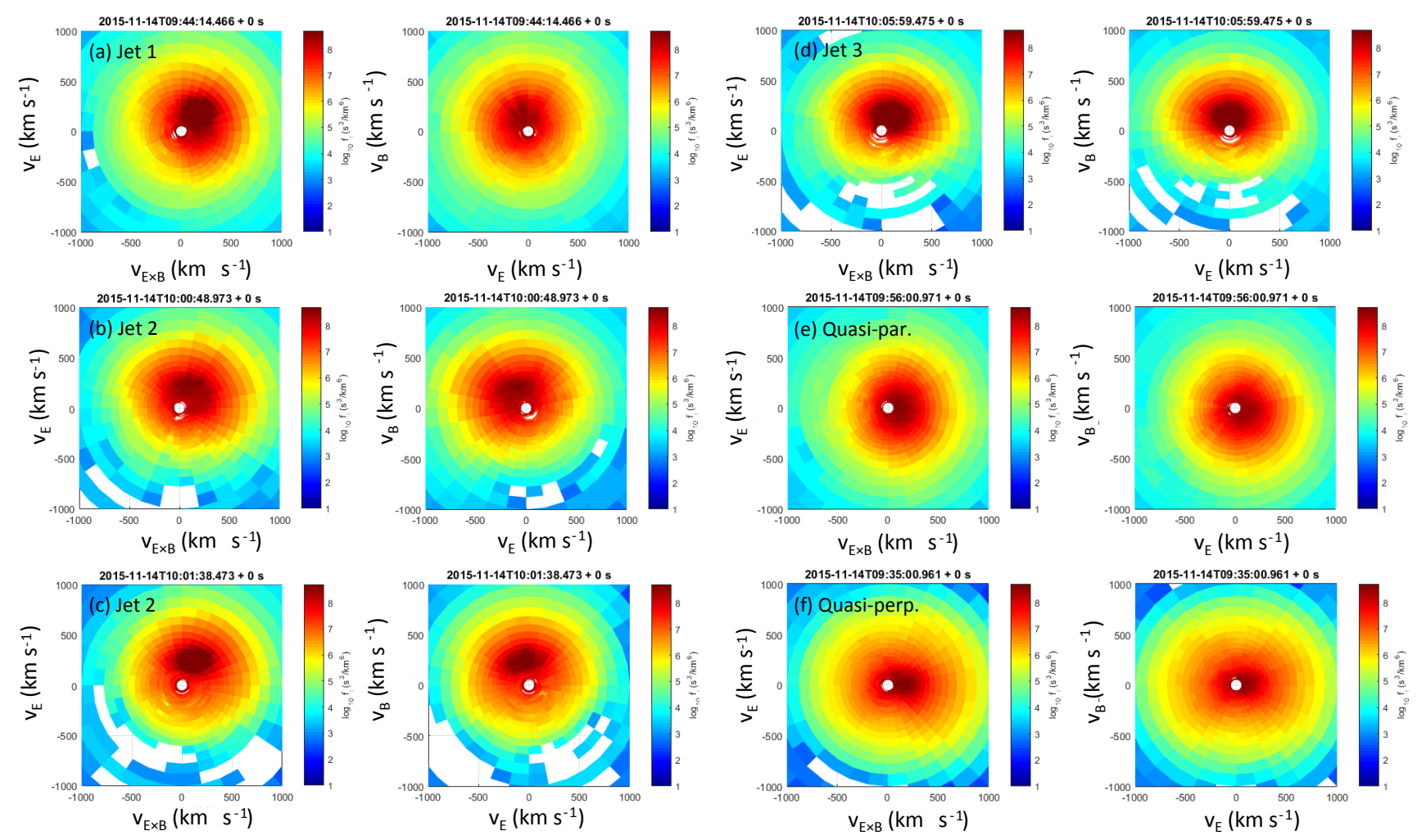

Figure 4. Ion distribution functions in the planes formed by the direction of the local magnetic field $\boldsymbol{B}$, and $\boldsymbol{E} \times \boldsymbol{B}$ and $\boldsymbol{E} \perp$, respectively. Distributions are shown for jets no. 1-3, and from the quasi-parallel and quasi-perpendicular magnetosheath outside of jet events.

is colder than the surrounding plasma, at both the leading and the trailing edge.

Finally, jet no. 3 is associated with an ion distribution with has a lower temperature than that of the surrounding quasiperpendicular magnetosheath. It also has a clear temperature anisotropy, which, however, is smaller than that of the surrounding plasma.

\subsubsection{Magnetic field configuration}

Recently both observations and simulations have shown that the magnetosheath jets may be related to a local deformation or stretching of the magnetic field (Plaschke et al., 2017; Karimabadi et al., 2014). Strongly curved magnetic field lines could be associated with magnetic forces accelerating or braking the jets, as they propagate in the magnetosheath. The magnetic field configuration could also have an effect on the interaction of the jet with the magnetopause. In Fig. 5 we investigate the magnetic field configuration of the jets under study here. Apart from the dynamic pressure and ion velocity, we show the ratio between the perpendicular to parallel ion velocity. Here the directions are relative to the magnetic field, and both the velocity ratio and the magnetic field are smoothed with a $120 \mathrm{~s}$ window in order to remove smallscale variability, waves and turbulence. (The $120 \mathrm{~s}$ window was chosen by trial and error to bring out the large-scale vari- ations that can be discerned in Fig. $5 \mathrm{~d}$ and $\mathrm{f}$, as well as considering the typical periods of up to $40 \mathrm{~s}$ of waves generated in the foreshock, e.g. Eastwood et al., 2005; Archer et al., 2012. This is also similar to the $60 \mathrm{~s}$ window used by Archer and Horbury, 2013 with the same motivation.) We also show the magnetic field components and magnitude, both with and without application of a smoothing window.

While the ion velocity is consistently perpendicular to $\boldsymbol{B}$ before the observations in the quasi-parallel magnetosheath, and more parallel to it afterwards, within the quasi-parallel magnetosheath there is a large variability in the velocity ratio, with no clear correlation to variations in $P_{\text {dyn }}$.

Jet no. 2 is clearly associated with a magnetic field discontinuity apparent in the rapid change of $B_{y}$ (most clearly seen in Fig. 5e). Most of the change is associated with the leading edge of the jet, which also marks the end of the isotropic, suprathermal plasma population. For jet no. 3 no apparent magnetic field discontinuity can be identified, while for no. 1 the situation is more unclear. There are several relatively sharp changes in the magnetic field, but it is difficult to unambiguously relate any of them to the jet.

Jets no. 2 and 3 show rather clear increases in $|\boldsymbol{B}|$ correlated with the increases in $P_{\text {dyn }}$, as reported for some jets by Archer and Horbury (2013) and Plaschke et al. (2013), as well as for fast plasmoids by Karlsson et al. (2015b). For jet 


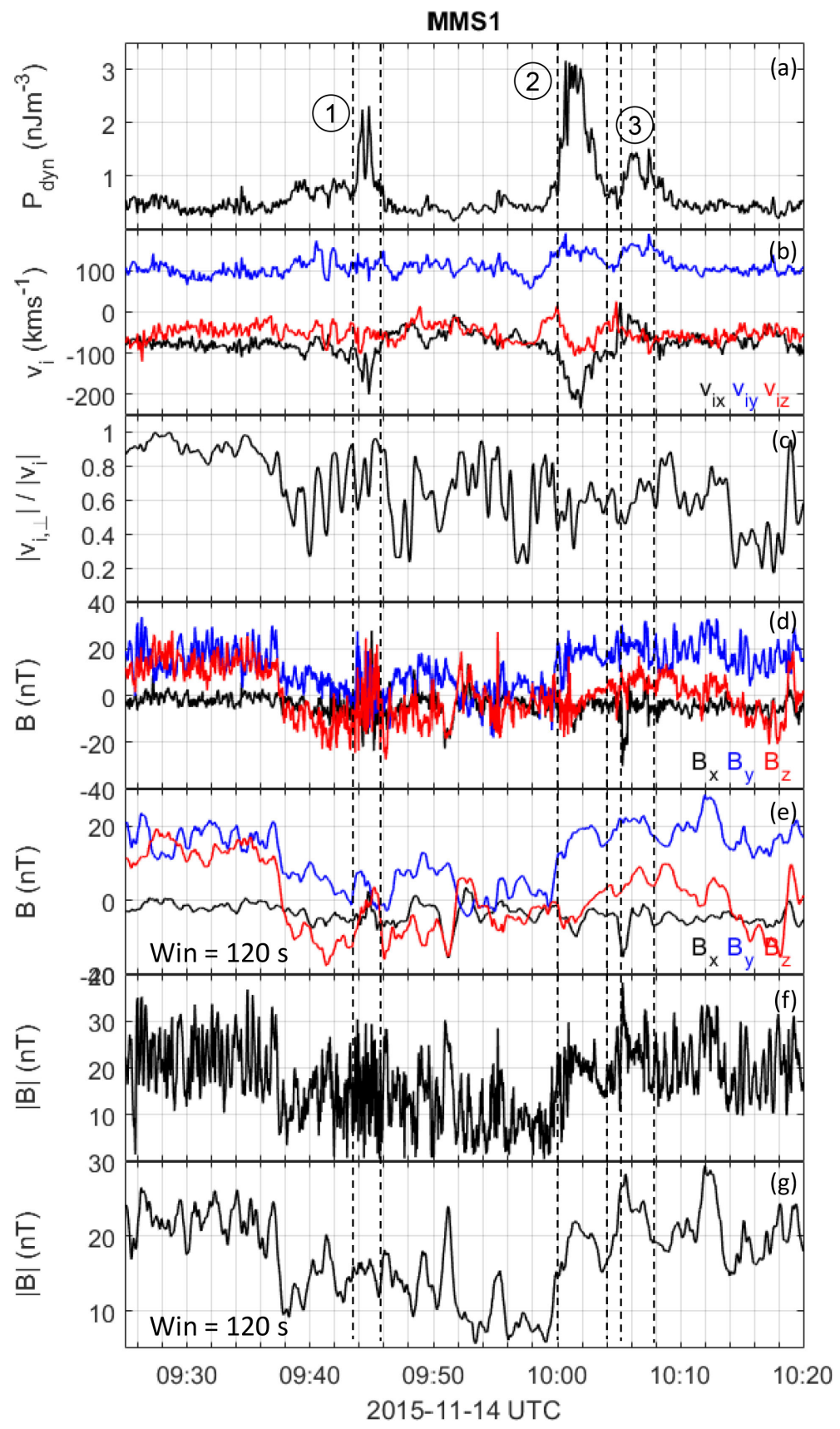

Figure 5. Magnetic field configuration. From top to bottom: dynamic pressure, ion velocity, ratio of perpendicular velocity to absolute velocity, magnetic field, magnetic field smoothed with a $120 \mathrm{~s}$ window, magnitude of magnetic field, and magnitude of magnetic field smoothed with a $120 \mathrm{~s}$ window.

no. 1 no clear increase in the magnetic field strength can be seen, although larger-amplitude, low-frequency wave activity is observed (panels $\mathrm{d}$ and f). This will be discussed below.

\subsubsection{Waves}

The quasi-parallel magnetosheath typically exhibits largeamplitude, low-frequency waves, that may originate either from the upstream solar wind and foreshock, or from local instabilities (e.g. Lucek et al., 2005, and references therein). In this section we will investigate wave emissions associated with the jets in the frequency range of $0.05 \mathrm{~Hz}-30 \mathrm{kHz}$, and compare them to the general wave activity in the surrounding magnetosheath.

Figure $6 \mathrm{~b}$ and $\mathrm{c}$ show MMS1 medium-frequency power spectral density (PSD) from the on-board spectrograms, summed over all three components for both $\boldsymbol{E}$ and $\boldsymbol{B}$ (re- 


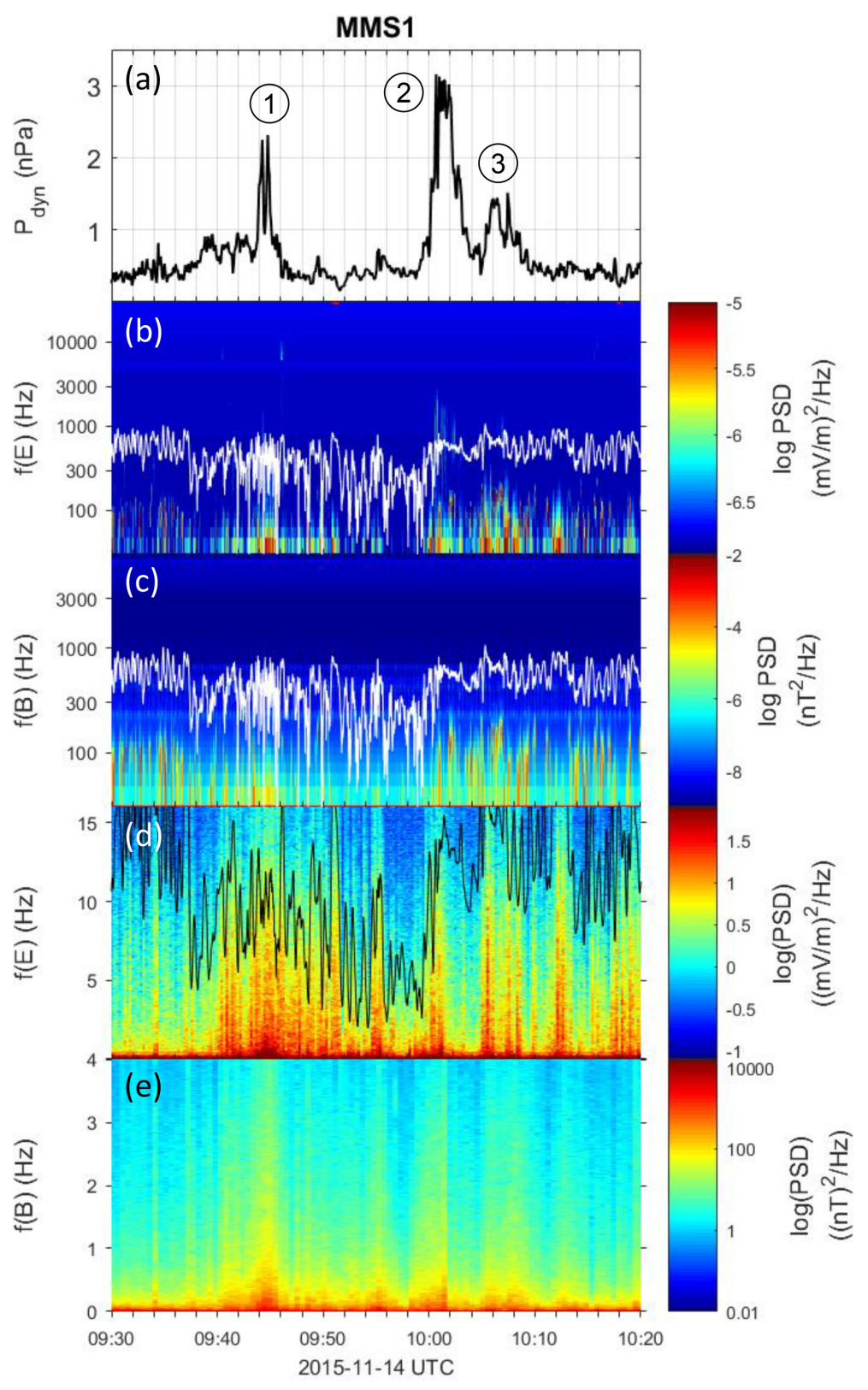

Figure 6. High- and medium-frequency wave activity. From top to bottom: dynamic pressure, on-board omnidirectional electric and magnetic field power spectral density (with the electron cyclotron frequency indicated by the white line), omnidirectional electric and magnetic field constructed from the survey and slow survey data, respectively. For the electric field spectrogram, the local lower-hybrid frequency is indicated by the black line.

ferred to as the "omnidirectional PSD"), with the electron gyrofrequency $f_{\text {ce }}$ indicated. Over most of the interval shown there are emissions below about $0.5 f_{\text {ce }}$ both in $\boldsymbol{E}$ and $\boldsymbol{B}$, but with a tendency for larger power spectral densities associated with the jets. This is consistent with the findings of Gunell et al. (2014), who interpreted these emissions as electron whistler waves. However, we can note that there are also some weak electrostatic oscillations extending above the electron gyrofrequency for jet no. 2 .
Figure 6d-e show omnidirectional spectrograms constructed from the waveform data of $\boldsymbol{E}$ and $\boldsymbol{B}$. There are increases in the power spectral density at and below the hydrogen lower hybrid frequency for $\boldsymbol{E}$, and also for $\boldsymbol{B}$, particularly at lower frequencies. The levels of wave activity are higher close to the jets, in particular at the edges of the jets. Again, the behaviour is similar to that reported by Gunell et al. (2014). 


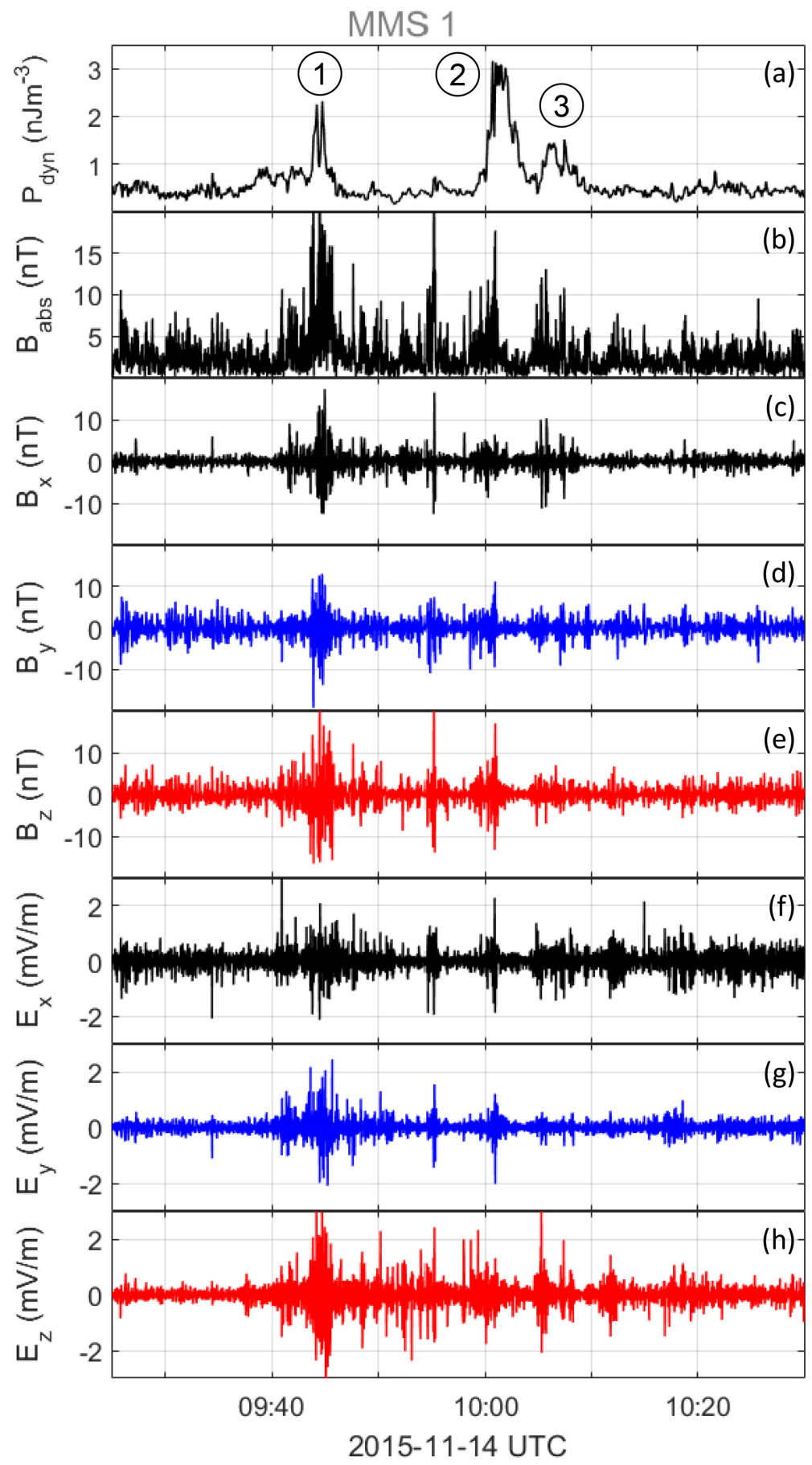

Figure 7. Low-frequency wave activity. From top to bottom: dynamic pressure, absolute value of magnetic field, and magnetic and electric field components. For all panels (except the top one) the DC component has been removed by subtracting a $25 \mathrm{~s}$ running average.

Figure 6 indicates that there is considerable wave activity at frequencies close to and below $1 \mathrm{~Hz}$. In order to study this in more detail, we plot in Fig. 7 the electric and magnetic field wave forms in the frequency range $0.04-8 \mathrm{~Hz}$, for the time interval 09:25-10:30 UTC. The upper frequency limit is given by the magnetic field sampling rate. The lower limit is chosen to suppress the DC component, and is implemented by the subtraction of a sliding window average. The electric field is also resampled to the $8 \mathrm{~Hz}$ magnetic field sampling rate.

Inside the quasi-parallel magnetosheath, at approximately 09:37-10:00 UTC, there is a general weak increase in the 
fluctuations in the magnetic field, with a compressional component. The fluctuation level is markedly increased inside jet no. 1 and at the leading edge of jet no. 2. Clear increases can be seen also at around 09:55 UTC, where a small increase in the dynamic pressure is observed, and collocated with jet no. 3, although this is outside of the general quasi-parallel magnetosheath region. The electric field shows a similar behaviour.

A more detailed look at jet no. 1 in Fig. 8a shows quasiperiodic oscillations in $\boldsymbol{B}$ and $\boldsymbol{E}$, without any appreciable phase shifts between the two fields. As a consequence, the Poynting flux $(\boldsymbol{S})$ has a relative well-defined direction over the whole interval, and points in the same general direction as the plasma flow inside the jet, i.e. with comparable $x$ and $y$ components.

In Fig. 8b, it is clear that the large-amplitude fluctuations are concentrated at the leading edge of jet no. 2 , where the ion distribution is more isotropic and similar to the quasi-parallel magnetosheath. In this region the Poynting flux has a similar direction to that in jet no. 1 , although with somewhat smaller values. At the trailing edge of the jet, where the ions are more anisotropic, the fluctuations have a much smaller amplitude. Finally, in jet no. 3, the fluctuations are similar to those in jet no. 1 and the leading edge of no. 2. However, the Poynting flux has an appreciably smaller $x$ component. We therefore conclude that the Poynting flux within the jets (when it has a large amplitude) points in the general direction of the plasma flow within the jets.

\subsubsection{Forces acting on the jet plasma}

Not much is known about the evolution of magnetosheath jets, as they move downstream from the bow shock. The plasma inside the jets may be either accelerated or slowed down by forces acting on it. The main candidate forces would be either the $\boldsymbol{j} \times \boldsymbol{B}$ force or the thermal pressure gradient force, $-\nabla p_{\text {th }}$. With the MMS spacecraft we can calculate these forces, which represent fluid effects. (When there is a large magnetic field gradient, non-fluid effects may be important, but away from the magnetopause the fluid effects will dominate.) Both the pressure gradient and the current density were calculated by the reciprocal vector method (Vogt et al., 2008). (For the determination of the current density, this method is equivalent to the curlometer method; Vogt et al., 2008.) The result is seen in Fig. 9. For the whole interval, the thermal pressure gradient force dominates the magnetic force. The latter is directed in the negative $x_{\mathrm{GSE}}$ direction over most of the quasi-parallel magnetosheath region. This pressure gradient force is consistent with a large-scale decrease in pressure as the spacecraft moves towards the bow shock, apparent when inspecting a larger time interval (not shown here). Note that some of the variations on a medium timescale may be of temporal nature. However, closer investigation would be necessary to exclude a systematic offset, although it is clear that there is no particular increase or de- crease in the pressure gradient force associated with the jets. The same is true for the magnetic force.

\subsection{Overview, 23 November 2015}

Before we begin to interpret the above observations and compare them to earlier results and theories, we should ask how typical they are. This is a question that should and will be addressed by a statistical investigation. In the meantime, we will present observations from another magnetosheath jet event (in slightly less detail) to show that the present observations are mostly not unique to this particular case. We will do this in the following section.

\subsubsection{Velocity, plasma properties, and forces}

Figure 10 shows a similar event to the one above. The observations were made when MMS1 was located at $\boldsymbol{r}_{\mathrm{GSE}}=(11.8,0.9,-0.8) R_{\mathrm{E}}$. A region characterized by the suprathermal ions typical of the quasi-parallel magnetosheath, seen in panel (e) between around 05:48:30 and 06:37 UTC, contains three regions of increased dynamic pressure, marked as jets no. 4-6 in panel (a).

Jets no. 5 and 6 have a very well-defined structure, with an isolated appearance in panel (a), while jet no. 4 is more complicated, with appreciable substructure. Jets no. 5 and 6 also have a clear density increase, collocated with the $P_{\text {dyn }}$ increase, and would be classified as fast plasmoids. Jet no. 4 has smaller density increases, which are also less correlated with $P_{\mathrm{dyn}}$, and would not be identified as a plasmoid.

Similarly to jet no. 2 of the first event, jet no. 6 , which is located at the trailing edge of the quasi-parallel magnetosheath region, is collocated with the boundary between the quasi-parallel and quasi-perpendicular magnetosheath, and is associated with a pronounced magnetic field discontinuity. It has a larger maximum in $P_{\mathrm{dyn}}$ than the other jets of this event, similar to the event from 14 November 2015. Spanning the boundary, it also contains two different plasma populations, with a low ion temperature anisotropy in the leading edge of the jet, overlapping the quasi-parallel magnetosheath, and a larger anisotropy in the trailing edge. Inspection of distribution functions (not shown here) confirms this and shows a very similar behaviour to that of jet no. 3 . While the temperature anisotropy is different in the leading and trailing edge, the temperature remains low over the whole jet.

Jets no. 4 and 5, however, have a low ion temperature anisotropy, comparable to the rest of the quasi-parallel magnetosheath. Also, for these jets, inspection of distribution functions show similar isotropic ion distributions as for jets no. 1 and 2. Jet no. 5 has an ion temperature which is clearly anti-correlated with the dynamic pressure. For jet no. 4 the temperature is generally depressed, but it is difficult to see a detailed anti-correlation with $P_{\text {dyn }}$.

The forces acting on the plasma are shown in panels (h) and (i) and exhibit a similar behaviour to the first 
(a)

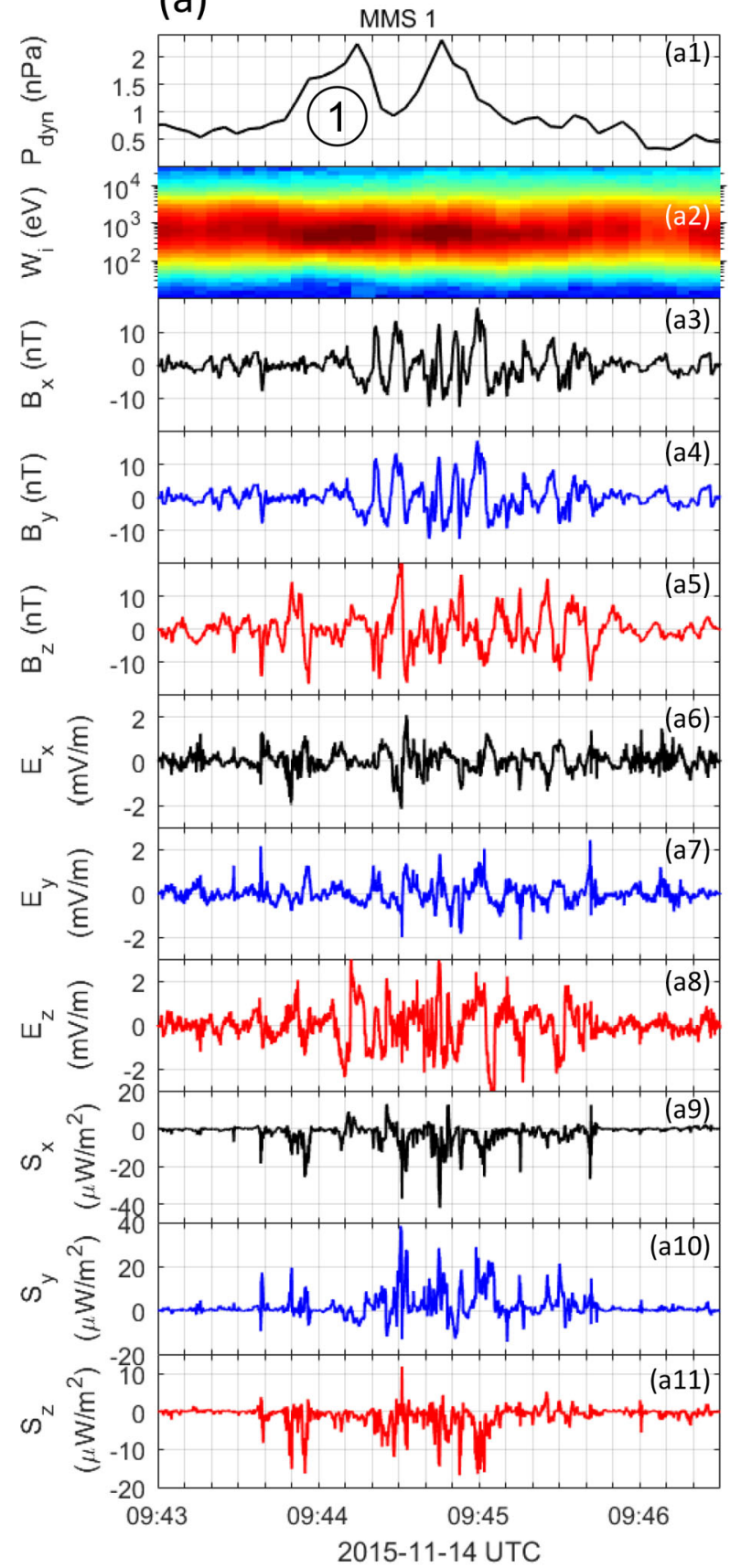

(b)

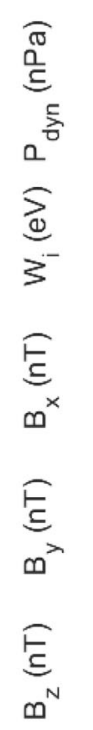

它
$\infty^{x}$
它
$\infty^{x}$
它
$\infty^{N}$

(b3)

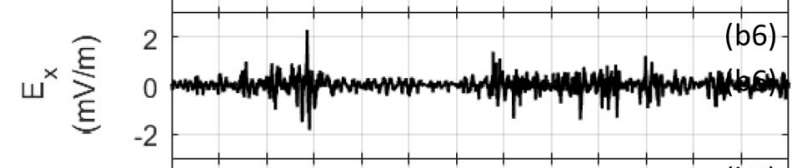

(b7)

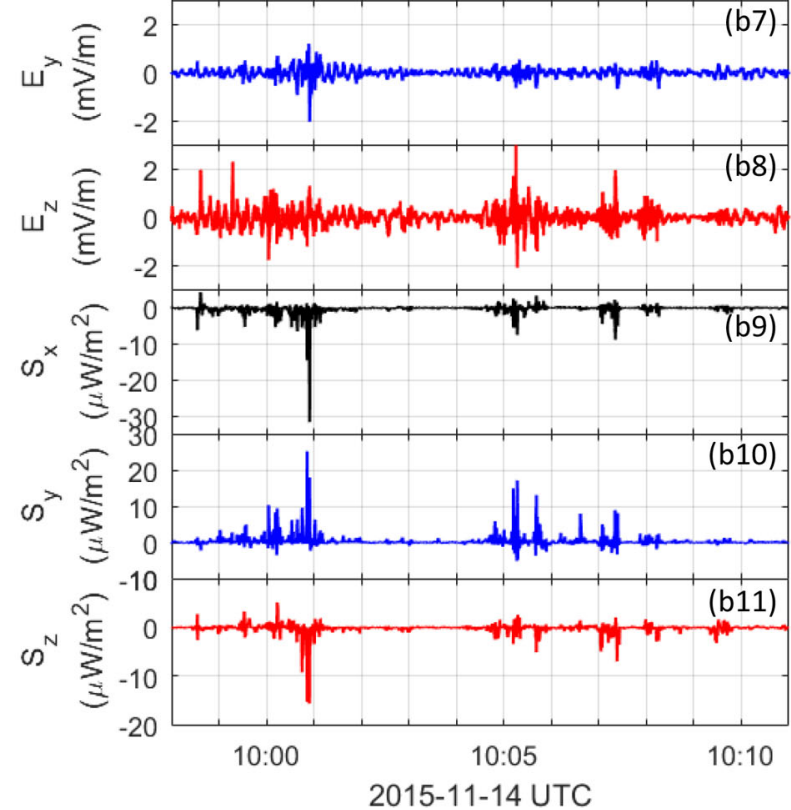

Figure 8. Detailed view of low-frequency wave activity for jets no. 1 (a) and jets no. 2 and 3 (b). From top to bottom: dynamic pressure, ion spectrogram, electric and magnetic fields with the DC component removed as in Fig. 7, and components of the Poynting flux $(\boldsymbol{S})$, calculated from the low-frequency electric and magnetic field shown in sub-panels (a3)-(a8) and (b3)-(b8), respectively.

event. The pressure gradient force is acting to break the magnetosheath plasma as it moves towards the magnetopause, with no clear correlation with the jets. There is some smallscale variation in the magnetic force within the quasi-parallel magnetosheath region; however, no clear signature related to the jets can be discerned.

\subsubsection{Magnetic field configuration}

In Fig. 11c we can see that for this event the velocity is consistently perpendicular to the magnetic field outside of the quasi-parallel magnetosheath region, while inside it there are large variations of the ratio $\frac{v_{\mathrm{i}, \perp}}{v_{\mathrm{i}}}$. For this event, there is a clear correlation between the dynamic pressure of jet no. 5 


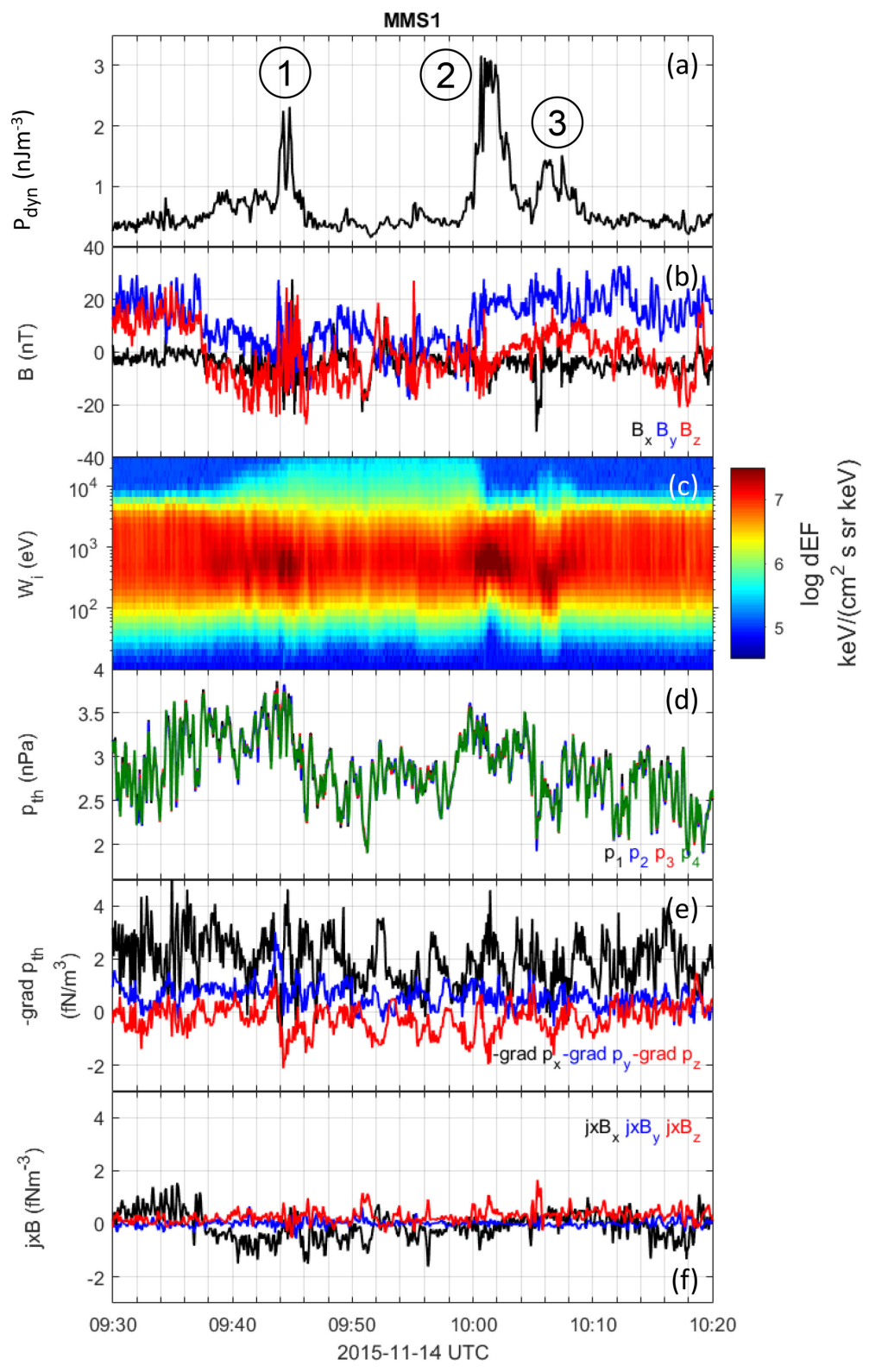

Figure 9. Forces on the jet plasma. From top to bottom: dynamic pressure, magnetic field, ion spectrogram, total thermal pressure for satellites 1-4, negative of the gradient of the total pressure, and the magnetic force $\boldsymbol{j} \times \boldsymbol{B}$.

and a clear decrease in $\frac{\boldsymbol{v}_{\mathrm{i}, \perp}}{\boldsymbol{v}_{\mathrm{i}}}$. For jet no. 6, the leading part is associated with a larger parallel velocity component than the trailing edge, where the velocity is purely perpendicular. For jet no. 3 no clear pattern can be discerned.

Jet no. 6 is clearly associated with a magnetic field discontinuity (Fig. 11d-g), which is collocated with the leading part of the jet and extends until the maximum in $P_{\text {dyn }}$ (and the ion velocity). The other jets are not associated with any magnetic field discontinuity.

Both jet no. 5 and 6 are associated with a relatively clear increase in the magnetic field magnitude (Fig. 11g), while it is difficult to see any clear correlation for jet no. 4 .

\subsubsection{Waves}

From Fig. 12a, we can see that there are emissions associated with the jets, which are likely associated with electron whistler waves (panels a2-a3) and lower hybrid waves (panel a4). In addition, however, there are strong electrostatic emissions extending up to a few kilohertz, far above the electron gyrofrequency (panel a2).

In Fig. 12b we see a similar behaviour as for the first event; large-amplitude, low-frequency variations in $\boldsymbol{E}$, and $\boldsymbol{B}$ with the largest Poynting fluxes collocated with the jets. Jet no. 4 , with the smallest $P_{\text {dyn }}$, also has the smallest Poynting fluxes. 


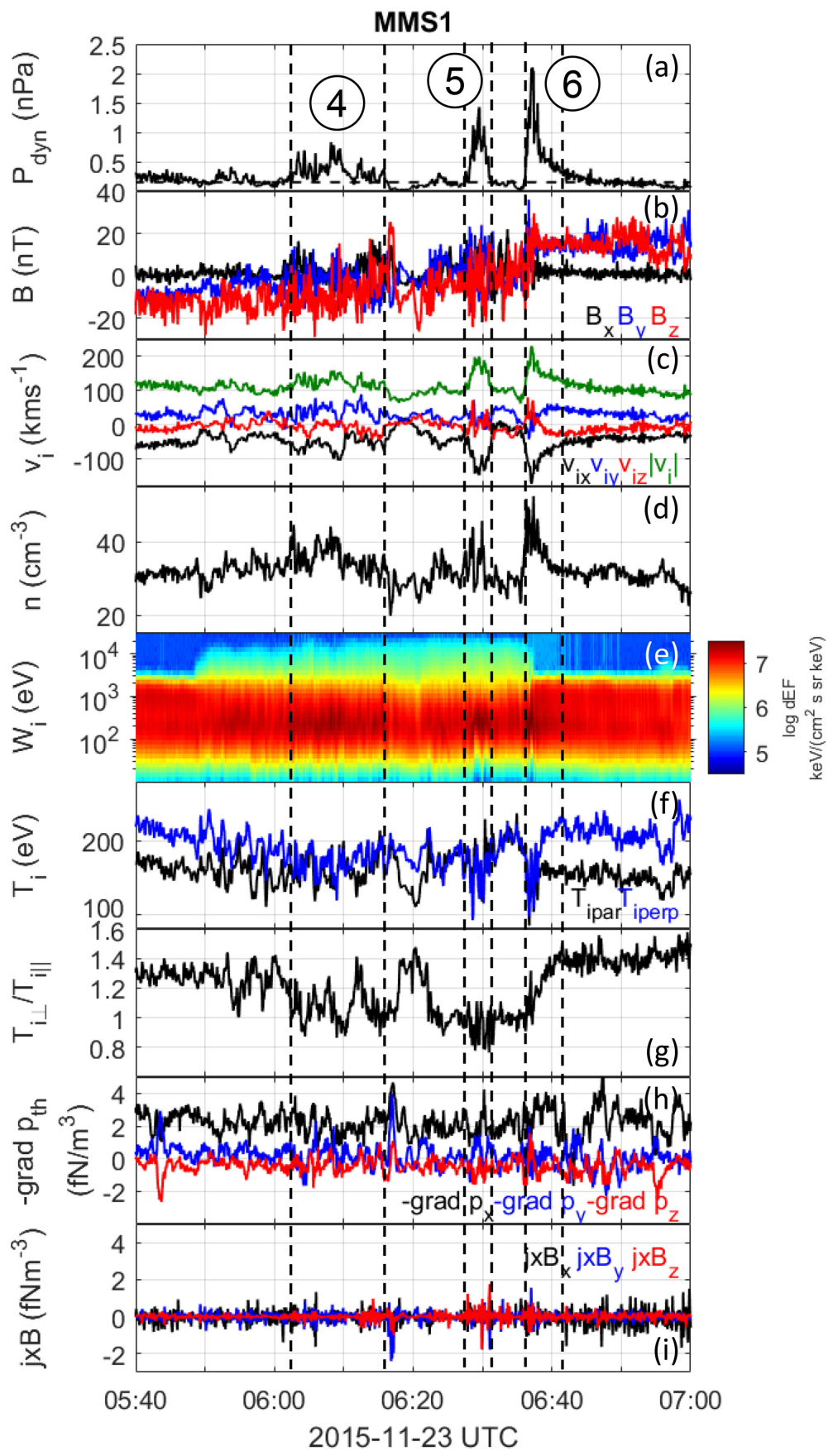

Figure 10. Overview of MMS1 measurements in the magnetosheath on 23 November 2015. From top to bottom are shown dynamic pressure, magnetic field $\boldsymbol{B}$, ion velocity $\boldsymbol{v}_{\mathrm{i}}$, ion and electron density $\left(n_{\mathrm{i}}\right.$ and $\left.n_{\mathrm{e}}\right)$, omnidirectional ion differential energy flux, ion and electron temperatures $\left(T_{\mathrm{i}}, T_{\mathrm{e}}\right)$, ion temperature anisotropy $\left(T_{\mathrm{iperp}} / T_{\mathrm{ipar}}\right)$, negative of the gradient of the total pressure, and the magnetic force $\boldsymbol{j} \times \boldsymbol{B}$. Three localized increases in dynamic pressure have been marked as jets no. 4-6.

For jet no. 5, with a velocity mainly in the negative $x$ direction, $S$ also points mainly in the $x$ direction. There is also an appreciable Poynting flux in the $y$ and $z$ directions, but it is distributed about equally in the two directions. For jet no. 4, which has an appreciable $v_{y}$ component, the Poynting flux is also more directed in the $y$ direction. This be- haviour is again consistent with a dominating emission of low-frequency waves in the same direction as the flow direction of the jets.

Some of the properties of jets no. 1-6 are summarized in Table 1. 


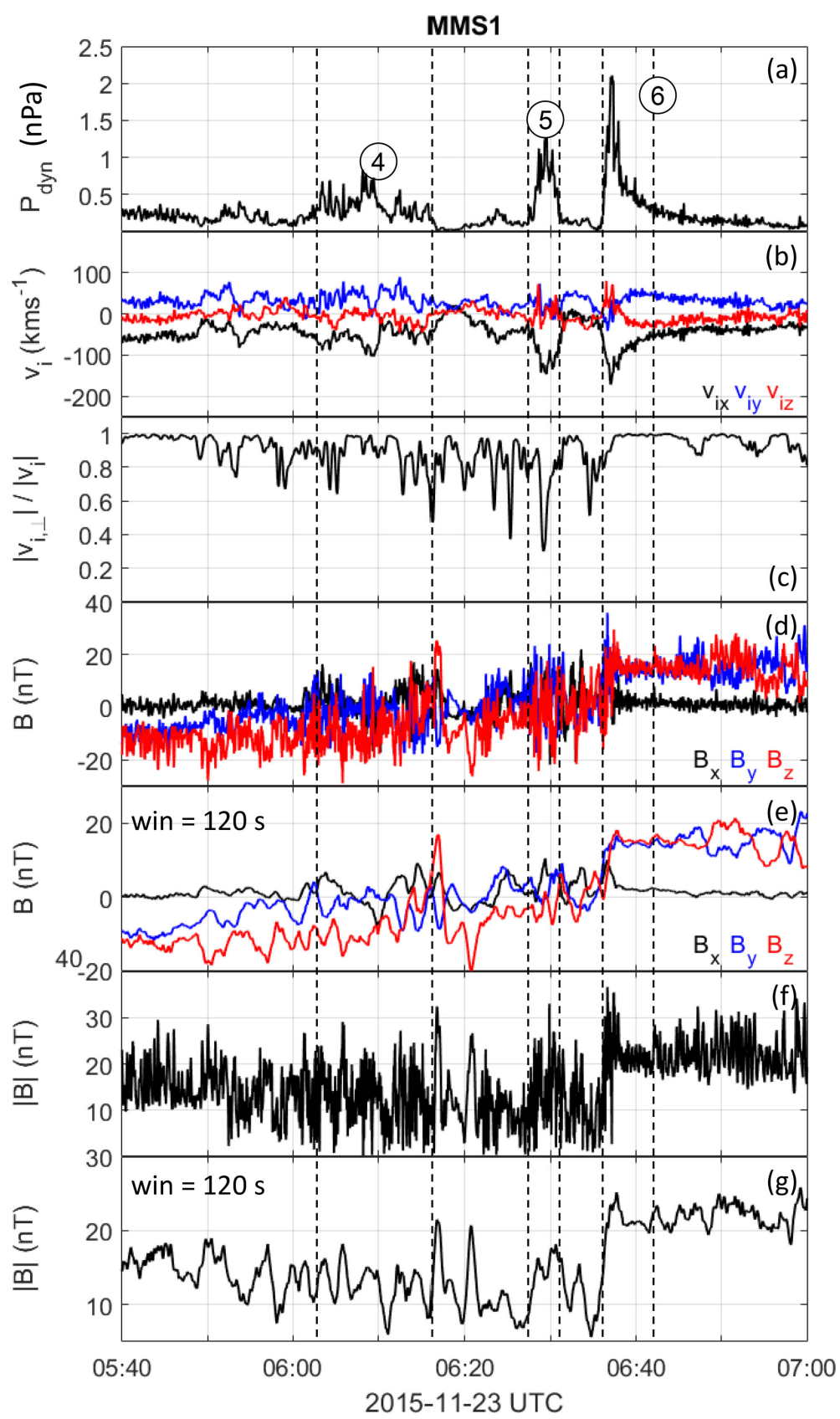

Figure 11. Magnetic configuration for jets on 23 November 2015, in the same format as Fig. 5.

\section{Interpretation and discussion}

From Figs. $1 \mathrm{~b}$ and 10 it appears that we have observed two quite distinctly different types of magnetosheath jets. Jets no. 2 and 6 are collocated with a magnetic field discontinuity that marks the boundary between the quasi-parallel and the quasi-perpendicular magnetosheath. Spanning this boundary, they contain two separate plasma populations corresponding to the two different magnetosheath regions. These jets have a higher dynamic pressure than that of the jets found inside the quasi-parallel magnetosheath, which is consistent with the findings of Archer et al. (2012) and the recent investigation of Plaschke et al. (2017).

Jets no. 1, 4, and 5, however, are embedded within the quasi-parallel magnetosheath and are not clearly associated with any magnetic field discontinuity. They contain only one type of plasma population, with an isotropic ion temperature, similar to that of the surrounding quasi-parallel magnetosheath.

Jet no. 3, which has likely interacted with the magnetopause, has similar properties to that of jets no. 1, 4, and 5, 

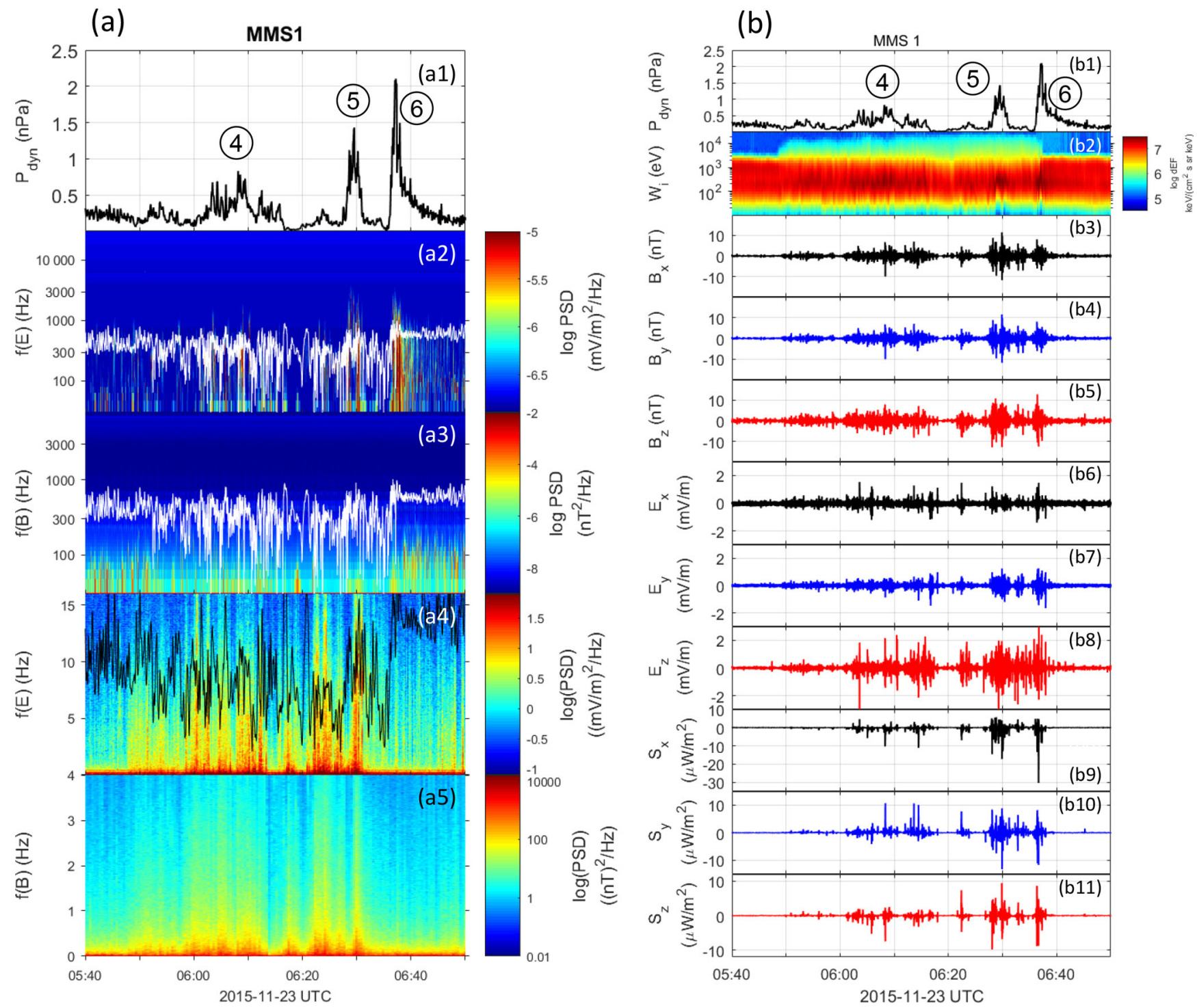

Figure 12. (a) High- and medium-frequency wave activity on 23 November 2015 in the same format as Fig. 6. (b) Low-frequency wave activity for the same time interval as in (a), and in the same format as Fig. 8.

but has a larger ion temperature anisotropy. We will briefly discuss this jet further below.

The different properties of the two types of jets may point to different generation mechanisms. We will first discuss the jets associated with a magnetic field discontinuity.

\subsection{Discontinuity-related jets}

Jets associated with magnetic field discontinuities have been suggested by Archer et al. (2012) to be generated when solar wind magnetic discontinuities interact with the bow shock. This was based on results from 1- and 2-dimensional hybrid simulations (Lin et al., 1996a, b), where the interaction of a rotational discontinuity with the bow shock resulted in density increases collocated with increased plasma flow velocity.
Together they constitute a pulse in the dynamic pressure. The increased flow velocity was produced via acceleration by the kinked magnetic fields resulting from the interaction between the rotational discontinuity and the bow shock.

There are, however, a few problems with applying the results of Lin et al. (1996a, b) to magnetosheath jets no. 2 and 6. First of all, the simulations produce structures which are very extended in the direction perpendicular to the magnetosheath flow. While the spacecraft separation of MMS is too small to determine the spatial scale of the jets, Archer et al. (2012) report scale sizes of $0.2-0.5 R_{\mathrm{E}}$ perpendicular to the average flow for jets associated with magnetic field discontinuities, and it is likely that the jets in this study have similar scale sizes. Plaschke et al. (2016) report on a median jet scale size perpendicular to the propagation direction 
Table 1. Summary of properties of the jets investigated.

\begin{tabular}{|c|c|c|c|c|c|c|c|}
\hline Jet no. & $\begin{array}{r}P_{\text {dyn, } \max } \\
(\mathrm{nPa})\end{array}$ & $\frac{P_{\mathrm{dyn}, \max }}{P_{\mathrm{dyn}, \mathrm{BG}}}$ & $T_{\mathrm{i}, \perp} / T_{\mathrm{i}, \|}$ & $\begin{array}{l}\text { Interacted } \\
\text { with MP }\end{array}$ & $\begin{array}{l}\text { B-field } \\
\text { discontinuity }\end{array}$ & $\begin{array}{l}\text { Field-aligned } \\
\text { flow }\end{array}$ & $\begin{array}{r}S_{\max } \\
\left(\mu \mathrm{W} \mathrm{m}^{-2}\right)\end{array}$ \\
\hline 1 & 2.3 & 6.4 & $0.8-1.1$ & No & No & No & 48 \\
\hline 2 & 3.2 & 8.9 & $\begin{array}{r}\text { Leading edge: } 1.0 \\
\text { Trailing edge: } 1.2-1.5\end{array}$ & No & Yes & No & 36 \\
\hline 3 & 1.5 & 4.2 & $1.2-1.6$ & Yes & No & No & 19 \\
\hline 4 & 0.8 & 4.0 & $0.9-1.4$ & No & No & No & 14 \\
\hline 5 & 1.4 & 7.0 & $0.8-1.1$ & No & No & Yes & 19 \\
\hline 6 & 2.1 & 10.5 & $\begin{array}{l}\text { Leading edge: } 1.0 \\
\text { Trailing edge: } 1.4\end{array}$ & No & Yes & No & 31 \\
\hline
\end{tabular}

of $0.9 R_{\mathrm{E}}$, although in their statistics jets with or without an association with a magnetic field discontinuity are not separated. These results, however, make it likely that jets at discontinuities have a limited perpendicular extension.

Furthermore, the pressure pulses of Lin et al. (1996b) have an anti-correlation between $P_{\text {dyn }}$ and $|\boldsymbol{B}|$, which is not consistent with the behaviour of jets no. 2 and 6 . Finally, the velocity increase in the simulations is mainly in the direction tangential to the bow shock surface, again in contradiction with the observed jets, which have their velocity increase mainly in the anti-sunward direction. The latter problem could, however, be partly resolved by considering magnetic field discontinuities with a normal not directed along the $x$ axis, but at some angle to that direction.

Lin et al. (1996b), however, report on another interesting phenomenon. When an interplanetary discontinuity is associated with a change of the bow shock configuration from quasi-parallel to quasi-perpendicular, the remaining foreshock plasma will be compressed against the now quasiperpendicular shock, and create a fast wave structure which will penetrate the bow shock. This fast wave structure will have a positive correlation between density and magnetic field and can catch up with the discontinuity further downstream of the shock, and possibly coalesce into a single structure (although it is unclear if the very sharp boundary between the two plasma populations within the jet would be retained in such a process). Since the foreshock already possesses small-scale variation in magnetic field and density with scale sizes of $0.2-3 R_{\mathrm{E}}$ (e.g. Schwartz and Burgess, 1991; Lucek et al., 2002), this could explain the limited extent of the jets. The higher velocity of the discontinuity jet compared to non-discontinuity-related jets would here be due to the higher velocity of the fast wave structure. A similar scenario was suggested by Dmitriev and Suvorova (2012). A schematic of this and the following scenarios can be found in Fig. 13 (panel a for this case).

This scenario could be tested by observations closer to the bow shock than those reported here. It also predicts that large increases in $P_{\mathrm{dyn}}$ should be more common at the trailing edge of a quasi-parallel magnetosheath region (where there is a change from a quasi-parallel to a quasi-perpendicular magnetosheath). While the two cases presented here are consistent with that prediction, Archer and Horbury (2013) and Plaschke et al. (2017) report on the largest $P_{\text {dyn }}$ located at the leading edge of the quasi-parallel magnetosheath. Furthermore, Plaschke et al. (2017) report on small-scale discontinuities at the leading edge of such a jet. It is unclear if these small-scale structures are important in understanding the jet formation, if they are created after it is formed, or if they are created in the foreshock and are advected by the jet plasma flow. In the latter cases their main importance will be in affecting the jet interaction with the magnetopause, as suggested by Plaschke et al. (2017). Obviously, more statistical investigations are needed, in particular with further burst measurements.

A remaining difficulty with this scenario is that jets no. 2 and 6 are associated with a clear decrease in both the perpendicular and parallel ion temperature. It is difficult to explain this other than as a signature of solar wind or foreshock plasma that is less processed by passing through the bow shock than the rest of the magnetosheath. This is one of the main predictions of the mechanism proposed by Hietala et al. $(2009,2012)$ and Hietala and Plaschke (2013). The temperature decrease is also the reason why solar wind interaction with hot flow anomalies is also an unlikely generation mechanism for these jets, as pointed out by Archer et al. (2012). The interaction of the discontinuity with bow shock corrugations also imposes a scale size of the order of $1 R_{\mathrm{E}}$ in the direction perpendicular to the jet velocity (Hietala et al., 2009).

The above scenario therefore needs to include an interaction with the bow shock according to the Hietala model, where the jet will pass the bow shock at local indentations, allowing the plasma to pass with much less deceleration and heating than the surrounding solar wind plasma. This is an alternative way of imposing a jet scale size perpendicular to the flow (see Fig. 13b).

Additional acceleration of the jet plasma could also conceivably result if the primed, stretched magnetic field lines, produced by the interaction of the discontinuity with the bow 
shock, could be turned around and be more aligned with the Sun-Earth line. One way this could happen is if part of the stretched field structure passes through a bow shock corrugation, and if the original normal to the discontinuity already has some angle to the $x$ direction. The $\boldsymbol{j} \times \boldsymbol{B}$ force observations show that there is no clear increase in the magnetic force associated with jets no. 2 and 6 . Since the jets are observed close to the magnetopause, however, the magnetic field tension may already have been spent. The increases in magnetic field and density may come about because the flow may contain a low-amplitude SLAMS, which has a small enough phase velocity that it convects with the solar wind flow, as has been suggested by Karlsson et al. (2015b). This configuration is obviously rather complicated and is maybe less likely. However, a future test will be to investigate the magnetic force at jets of this type closer to the bow shock. This scenario is illustrated in Fig. 13c.

\subsection{Non-discontinuity-related jets}

Turning to jets no. 1, 4, and 5, none of these are associated with a clear magnetic field discontinuity, or with a clear particle boundary. In that sense they are consistent with the model proposed by Hietala et al. (2009), which postulated that jets may be generated during times of a steady IMF, behind the quasi-parallel bow shock. Also consistent with this mechanism is the lower ion temperatures associated with these jets.

Jet no. 5 has a very well-defined shape with clear correlations between $\boldsymbol{v}_{\mathrm{i}}, n_{\mathrm{i}}$, and $\boldsymbol{B}$, whereas jets no. 1 and no. 4 exhibit more structure in $\boldsymbol{v}_{\mathrm{i}}$, and no correlations with $\boldsymbol{B}$. Jet no. 1 has a clear correlation between $\boldsymbol{v}_{\mathrm{i}}$ and $n_{\mathrm{i}}$, while no clear correlations can be seen for jet no. 4 . These different behaviours may be related to slightly different scenarios associated with the Hietala model. If the jet is associated with a single, rather isolated inclined portion of the magnetosheath, as in Fig. 13d, a single, well-defined jet will result. The clear increases in magnetic field and density may again be related to a low-amplitude SLAMS, convecting with the solar wind flow (Karlsson et al., 2015b).

An alternative scenario producing an increased plasma density and magnetic field strength is the focusing of fast plasma associated with a concave bow shock corrugation (Fig. 13e). Such a configuration may produce a local velocity minimum in the centre of the jet. Intriguingly jet no. 1 has just such a minimum in its centre. The association with this mechanism is, however, rather speculative, since the jet is located far downtail of the bow shock and has no clear associated magnetic field signature.

We also note that, in contrast, a convex corrugation would produce diverging plasma flows (Fig. 13f). A combination of several corrugations, both concave and convex, adjacently located on the bow shock may produce a region of several maxima in plasma flow, with and without associated density and magnetic field increases, such as the appearance of jet no. 4 .
Jets no. 1, 4, and 5 all contain a plasma with isotropic ion temperatures, similar to the surrounding quasi-parallel magnetosheath plasma, but cooler. This is consistent with the results reported by Plaschke et al. (2013) and Archer et al. (2012), and again shows that the jets contain less processed solar wind plasma than the surrounding magnetosheath, and that they are also not subject to any anisotropic heating as they travel downstream.

Jet no. 3, however, contains a cool plasma with a pronounced temperature anisotropy. The anisotropy is due to an increased perpendicular temperature, although not as high as in the surrounding quasi-perpendicular magnetosheath. It is likely that this perpendicular heating is associated with an interaction of the jet with the magnetopause. The anisotropic temperature may be due to a compression of the jet plasma, with associated adiabatic heating (see Lemaire, 1985; Roth, 1992; Voitcu and Echim, 2017).

\subsection{Magnetic field configuration}

Plaschke et al. (2017) have recently reported that jets may modify the magnetosheath magnetic field, stretching it out so that it becomes more aligned with the jet propagation direction. This has also been predicted in simulations by Karimabadi et al. (2014). We see little evidence of that here, except for in jet no. 5 where the ion flow becomes almost parallel to the magnetic field. There may be some logic to this: the magnetic signature of jets no. 2 and 6 is perhaps mostly determined by the discontinuity, jet no. 4 has a more complicated morphology, and jet no. 3 has suffered the complication of interacting with the magnetopause. Jet no. 5 has a clear and well-defined velocity increase, which may give a clear signature of frozen-in field lines aligned with the plasma flow. Jet no. 1 has a smaller velocity increase with respect to the surrounding plasma than jet no. 5, and may therefore not show a clear alignment of the field and the jet plasma flow direction. This subject warrants further study, since the magnetic field associated with a jet may be important for its magnetospheric consequences. The local variation in the magnetic field and plasma $\beta$ may, for example, trigger local reconnection when the jet encounters the magnetopause.

\subsection{Wave emissions}

For both dates examined, we observe several kinds of wave emissions. There are increased spectral densities at and below the local lower hybrid frequency, mainly in the electric field. The emissions are seen both inside and outside of the jets, but with a generally increased amplitude inside of them, in particular for jets no. 3 and 5, and the leading parts of jets no. 2 and 6. This is consistent with the reports of lowerhybrid wave emissions by Gunell et al. (2014), and we note that the values of the power spectral densities are comparable. 


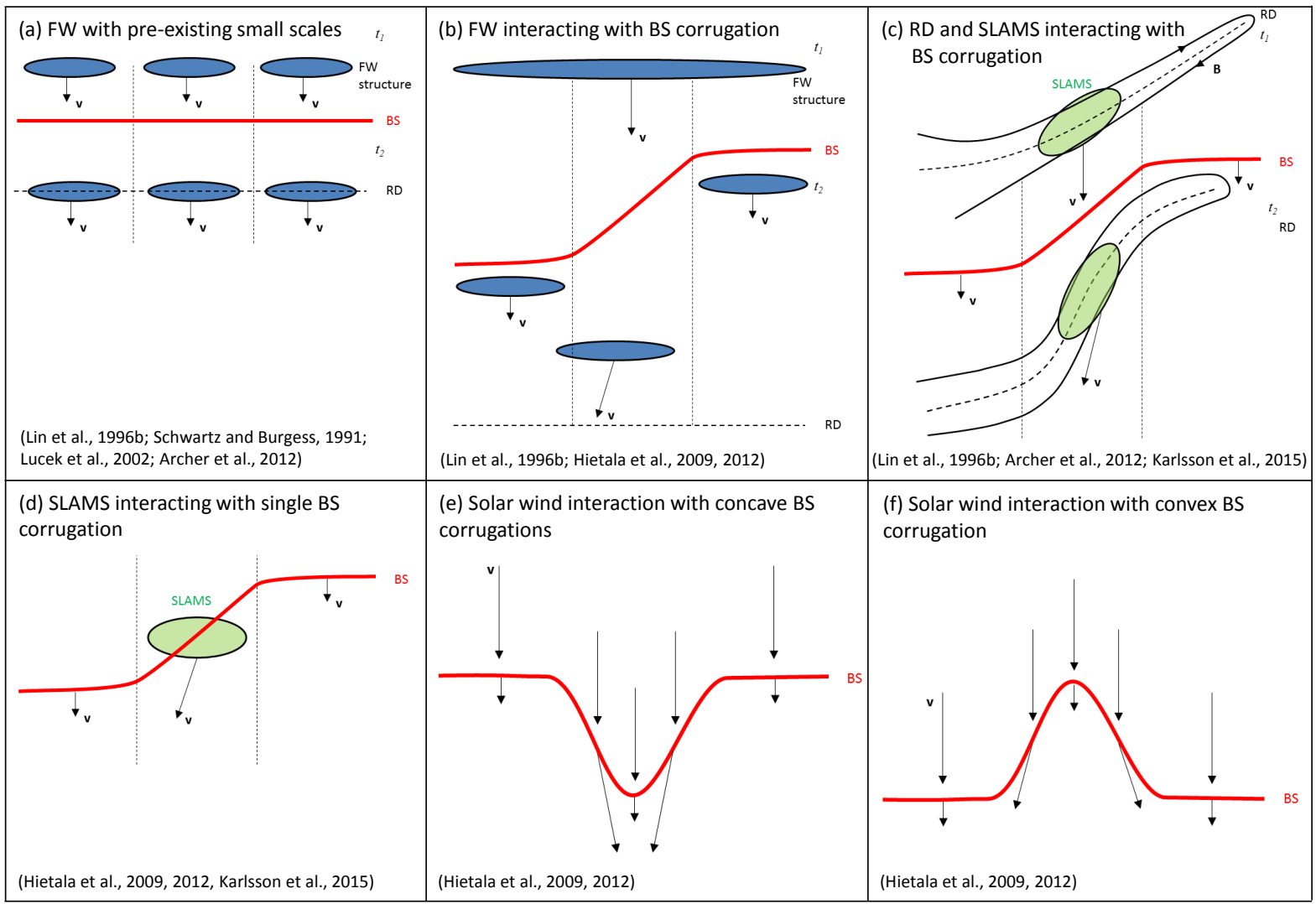

Figure 13. Six different scenarios for formation of the jets in this paper. $\mathrm{FW}=$ fast wave, $\mathrm{BS}=$ bow shock, $\mathrm{RD}=$ rotational discontinuity; $t_{1}$ and $t_{2}$ refers to different times. For each scenario the most relevant references are indicated in the respective panels.

Likewise, emissions at a few tens of percent of the local electron gyrofrequency, for both $\boldsymbol{E}$ and $\boldsymbol{B}$, were reported by the same authors, who interpreted them as whistler mode waves, based also on their propagation properties. Our observations are again consistent, as far as we can tell without the phase information.

Gunell et al. (2014) estimated electric and magnetic field energy densities associated with these waves, and found them in general to be lower than $10^{-12} \mathrm{Jm}^{-3}$. Since this is several orders of magnitudes smaller than the kinetic energy density (which is half of the dynamic pressure) of the jets reported on here, we conclude that the generation of such wave energy densities has no importance for the dynamics of the jets. For electromagnetic waves, however, more relevant is the amount of Poynting flux leaving the jet. No spectral phase information is available, but we can get an upper limit of the Poynting flux by assuming that all electric and magnetic field oscillations are in phase. We then get

$S \leq \frac{\sqrt{\int P_{E} \mathrm{~d} f \int P_{B} \mathrm{~d} f}}{\mu_{0}}$,

where $P_{E}$ and $P_{B}$ are the electric and magnetic field power spectral densities, and $f$ is the frequency, and we integrate over frequencies with assumed whistler waves. An estimate of $S$ for jet no. 1 then yields $S \leq 5 \times 10^{-8} \mathrm{~W} \mathrm{~m}^{-2}$. To get an upper limit of the energy loss of the jet due to this Poynting flux we approximate the jet with a cylinder of length $v_{\mathrm{i} x, \max } \Delta t$, where $v_{\mathrm{i} x, \max }$ is the maximum ion velocity of the jet in the $x$ direction, and $\Delta t$ its approximate lifetime. We also assume a radius of $1 R_{\mathrm{E}}$ (e.g. Plaschke et al., 2016). Assuming the estimated Poynting flux is everywhere normal to the boundary of the surface of the cylinder (to get an upper estimate) we get a total outflow of electromagnetic energy of around $2 \times 10^{7} \mathrm{Js}^{-1}$. Estimating the total kinetic energy of the jet by assuming a kinetic energy density of $1 \mathrm{nJm}^{-3}$, it would take approximately $22 \mathrm{~h}$ to radiate away the same amount of energy. Since this time is very long compared to the travel time for the jet to the magnetopause, we conclude that radiation losses by these waves are also likely to be unimportant for the evolution of the jets.

The generation of these waves will not be studied further here, but will be the subject of future work. However, we note that Gunell et al. (2014) suggests that the lower-hybrid waves are generated at the density gradients at the edges of the jets, and may propagate into the jet. Lower-hybrid wave generation at density gradients due to the lower hybrid drift instability is a well-established phenomenon in many differ- 
ent space plasmas (e.g. Huba et al., 1978; Gary and Eastman, 1979; André et al., 2001; Norgren et al., 2012; Karlsson et al., 2017). We also note that the whistler-type waves occur in short bursts, similar to the observations by Gunell et al. (2014), who speculate that this may be due to parallel electric fields set up at the jet boundary, accelerating electrons which emit the waves. Observations at higher time resolution may shed light on this.

A new result here, compared to the study by Gunell et al. (2014), is the presence of electrostatic emissions extending above the electron gyrofrequency. The energy densities are still many orders of magnitude smaller than the kinetic energy density, so the waves are not energetically important. We will again defer closer study of these waves to the future, but we can speculate that they are related to the broadband electrostatic noise (BEN) observed closed to the bow shock by, for example, Cattell et al. (2003) and Pickett et al. (2003), extending to frequencies between the electron cyclotron and plasma frequencies. The BEN may be non-linear, solitary structures associated with either ion acoustic or Langmuir waves.

A further new result is the existence of low-frequency, quasi-periodic electromagnetic oscillations, with higher amplitudes within the jets than in the surrounding quasi-parallel magnetosheath. Here we have direct observations of Poynting flux. The highest values of these fluxes are of the order of $50-60 \mu \mathrm{W} \mathrm{m}{ }^{-2}$, almost 2 orders of magnitude larger than that of the whistler-like waves. Since the Poynting flux is closely aligned with the jet propagation direction, we estimate the emitted electromagnetic energy by multiplying $\sqrt{S_{x}^{2}+S_{y}^{2}}$ with the surface area of the end of the cylinder (assuming that it is oriented with its axis parallel to the jet velocity), with the same dimensions as above. This yields a value of $8 \times 10^{9} \mathrm{~W}$, which gives a time of $7 \mathrm{~min}$ for radiating away all the kinetic energy of the jet, if they radiate with a constant value of the Poynting flux. This is a coarse estimate, but it shows that the low-frequency waves may be important to take into account when determining the evolution of the jets, similar to the results for bursty bulk flows in the inner magnetosphere (Stawarz et al., 2015).

ULF wave activity in the magnetosheath is often attributed to convection of waves generated in the foreshock or at the bow shock (Lucek et al., 2005). The fact that the lowfrequency waves are only present when the jets contain quasi-parallel magnetosheath-like plasma, and not in the trailing edges of jets no. 2 and 6, their approximate period of $\sim 10 \mathrm{~s}$ and the large compressional component hint at a relation to the so-called "10 s waves" (Hoppe and Russell, 1983; Eastwood et al., 2003). These are usually observed in the upstream foreshock, and are believed to be generated by an instability associated with diffuse ions in the foreshock (e.g. Blanco-Cano and Schwartz, 1997; Wilson, 2016).

Diffuse ions are the hot, isotropic part of the suprathermal ions of the foreshock and quasi-parallel magnetosheath (Tsu- rutani and Rodriguez, 1981). This is consistent with the increased wave amplitudes associated with the jets, which may contain relatively unprocessed foreshock plasma. Such a hot ion distribution can be observed in the ion distribution function from jet no. 1 , which is also associated with the largest wave amplitudes. In contrast, no sign of a counter-streaming ion population can be found, which is another source for ULF waves in the foreshock (Wilson, 2016). Such ion populations could conceivably result from a mixing of the jet plasma and the background magnetosheath plasma, but this evidently does not take place here.

If the diffuse ions are responsible for the generation of the low-frequency waves in the jets, the waves will not dissipate the kinetic energy of the jets, and may not influence their propagation. However, they may play an important role in thermalizing the magnetosheath plasma associated with jets.

\subsection{Forces}

We have already discussed the fact that no clear signature in the magnetic force could be found for the jets associated with the magnetic field discontinuities. No such signatures can be seen for any of the other jets either. The same is true for the thermal pressure gradients. We conclude that for the jets studied here, no appreciable acceleration or braking due to such forces take place close to the magnetopause. This is in contrast to forces acting on fast flows (bursty bulk flows) in the magnetotail, where Karlsson et al. (2015a) showed that the magnetic force brakes the bursty bulk flow plasma in the inner magnetosphere, while further downtail there is an acceleration due to the magnetic field tension force. An ongoing investigation of MMS measurements of bursty bulk flows also shows that localized ion thermal pressure gradients may brake the bursty bulk flows in the inner magnetosphere, in contrast to what the case is here.

\section{Summary and conclusions}

We have used MMS data to carry out a detailed analysis of the large- and meso-scale properties of magnetosheath jets with a modern suite of plasma instruments, based on the current understanding of such jets. We have studied six different magnetosheath jets during two time periods in November, 2015, and have made the following conclusions:

1. We have found that there are at least two distinct types of jets, with some clearly different properties.

a. Two of the jets are associated with a magnetic field discontinuity and straddle the boundary between the quasi-parallel and quasi-perpendicular magnetosheath. The different parts of the jets, therefore, contain different plasma populations: one isotropic ion population and another with a larger ion temperature anisotropy. Both ion populations are, however, cooler than the surrounding magnetosheath 
plasma. This type of jet had a larger maximal dynamic pressure than the second type, and although it may be more rare than the second one, it may therefore have a greater impact on the magnetopause interaction. The dynamic pressure is correlated with both velocity and density, as well as with magnetic field strength.

b. The second type is found embedded within the quasi-parallel magnetosheath, has no clear association with a magnetic field discontinuity, and contains a plasma with an isotropic ion distribution similar to the surrounding plasma but considerably cooler. This type of jet can either have a simple, well-defined structure, in which case it has a clear correlation with increases in plasma density and magnetic field strength, or a more complicated morphology, with a more unclear relation to density and magnetic field increases.

One jet that contained a quasi-parallel magnetosheath ion population, and therefore probably was an example of the second type, was found outside of the quasi-parallel magnetosheath. It is likely to have interacted with the magnetopause, and showed a weak ion anisotropy, possibly as the result of adiabatic heating.

2. All the jets studied here were associated with mediumand high-frequency wave emissions in the lower hybrid and whistler frequency ranges, as well as broadband electrostatic waves. None of these wave emissions were found to be energetically important, and they are therefore not likely to affect the evolution of the jets.

3. The isotropic ion populations within the jets were found to be associated with low-frequency electromagnetic waves with a compressional component. These waves had a Poynting flux of up to $40 \mu \mathrm{W} \mathrm{m} \mathrm{m}^{-2}$ and may be energetically important, depending on their generation mechanism.

4. A majority of the jets showed no clear field-aligned flow of the type reported by Plaschke et al. (2017) and found in simulations by Karimabadi et al. (2014), with the exception of jet no. 5 .

5. No clear signatures of magnetic or thermal pressure gradient forces were seen in association with the magnetosheath jets investigated here.

We have suggested that the two different types of jets are associated with different generation mechanisms, and have discussed different scenarios for their creation. The different nature of these types of jets suggests that future simulations and modelling of jets should address both types. In particular, 3-dimensional simulations may reveal new insights into the jet generation mechanisms for both types of jets. Likewise, separating the two different types in future statistical studies may help to reveal further differences in their properties, such as scale sizes, occurrence, geoeffectiveness (Plaschke et al., 2016), etc.

The wave emissions associated with the jets will be the subject of future study, in particular the role of the lowfrequency waves, both in terms of affecting the evolution of the jets, and influence on the magnetopause. Also, the relation between the flow velocity of the jets and the magnetic field configuration, where no consistent picture has emerged, will have to be the subject of further study. This may be of importance for the way magnetic forces may influence the dynamics of the jets in the magnetosheath, and the interaction of the jets with the magnetopause.

Data availability. The MMS data used in this study are available at the project home page https://lasp.colorado.edu/mms/sdc/ public/ (LASP, 2018). The THEMIS data and analysis software (SPEDAS) are available from http://themis.ssl.berkeley. edu (THEMIS, 2018).

Competing interests. The authors declare that they have no conflict of interest.

Acknowledgements. We acknowledge valuable discussions within the International Space Science Institute (ISSI), Bern, team 350 "Jets downstream of collisionless shocks", led by Ferdinand Plaschke and Heli Hietala. We acknowledge NASA contract NAS5-02099 and Vassilis Angelopoulos for use of data from the THEMIS Mission. Specifically we thank Karl-Heinz Glassmeier, Hans-Ulrich Auster and Wolfgang Baumjohann for the use of FGM data provided under the lead of the Technical University of Braunschweig and with financial support through the German Ministry for Economy and Technology and the German Center for Aviation and Space (DLR) under contract 50 OC 0302. The work of Heli Hietala is supported by NASA grant NNX17AI45G and contract NAS502099.

The topical editor, Georgios Balasis, thanks two anonymous referees for help in evaluating this paper.

\section{References}

Amata, E., Savin, S. P., Ambrosino, D., Bogdanova, Y. V., Marcucci, M. F., Romanov, S., and Skalsky, A.: High kinetic energy density jets in the Earth's magnetosheath: A case study, Planet. Space Sci., 59, 482-494, https://doi.org/10.1016/j.pss.2010.07.021, 2011.

André, M., Behlke, R., Wahlund, J.-E., Vaivads, A., Eriksson, A.-I., Tjulin, A., Carozzi, T. D., Cully, C., Gustafsson, G., Sundkvist, D., Khotyaintsev, Y., Cornilleau-Wehrlin, N., Rezeau, L., Maksimovic, M., Lucek, E., Balogh, A., Dunlop, M., Lindqvist, P.-A., Mozer, F., Pedersen, A., and Fazakerley, A.: Multi-spacecraft observations of broadband waves near the lower hybrid frequency at the Earthward edge of the magnetopause, Ann. Geophys., 19, 1471-1481, https://doi.org/10.5194/angeo-19-1471-2001, 2001. 
Archer, M. O. and Horbury, T. S.: Magnetosheath dynamic pressure enhancements: occurrence and typical properties, Ann. Geophys., 31, 319-331, https://doi.org/10.5194/angeo-31-319-2013, 2013.

Archer, M. O., Horbury, T. S., and Eastwood, J. P.: Magnetosheath pressure pulses: Generation downstream of the bow shock from solar wind discontinuities, J. Geophys. Res., 117, A05228, https://doi.org/10.1029/2011JA017468, 2012.

Archer, M. O., Hartinger, M. D., and Horbury, T. S.: Magnetospheric "magic" frequencies as magnetopause surface eigenmodes, Geophys. Res. Lett., 40, 5003-5008, https://doi.org/10.1002/grl.50979, 2013a.

Archer, M. O., Horbury, T. S., Eastwood, J. P., Weygand, J. M., and Yeoman, T. K.: Magnetospheric response to magnetosheath pressure pulses: A low-pass filter effect, J. Geophys. Res., 118, 5454-5466, https://doi.org/10.1002/jgra.50519, 2013b.

Baker, D., Riesberg, L., Pankratz, C., Panneton, R., Giles, B., Wilder, F., and Ergun, R.: Magnetospheric multiscale instrument suite operations and data system, Space Sci. Rev., 199, 545-575, 2016

Baumjohann, W. and Treumann, R. A.: Basic space plasma physics, World Scientific Publishing Company, London, UK, 2012.

Blanco-Cano, X. and Schwartz, S.: Kinetic theory mode properties: Application to low frequency waves in the ion foreshock, Adv. Space Res., 20, 707-711, https://doi.org/10.1016/S02731177(97)00460-2, 1997.

Burch, J., Moore, T., Torbert, R., and Giles, B.: Magnetospheric multiscale overview and science objectives, Space Sci. Rev., 199, 5-21, 2016.

Cattell, C., Neiman, C., Dombeck, J., Crumley, J., Wygant, J., Kletzing, C. A., Peterson, W. K., Mozer, F. S., and André, M.: Large amplitude solitary waves in and near the Earth's magnetosphere, magnetopause and bow shock: Polar and Cluster observations, Nonlin. Processes Geophys., 10, 13-26, https://doi.org/10.5194/npg-10-13-2003, 2003.

Chang, S.-W., Scudder, J., Fennell, J., Friedel, R., Lepping, R., Russell, C., Trattner, K., Fuselier, S., Peterson, W., and Spence, H. E.: Energetic magnetosheath ions connected to the Earth's bow shock: Possible source of cusp energetic ions, J. Geophys. Res.:-Space, 105, 5471-5488, 2000.

Chao, J., Wu, D., Lin, C.-H., Yang, Y.-H., Wang, X., Kessel, M., Chen, S., and Lepping, R.: Models for the size and shape of the Earth's magnetopause and bow shock, in: Cospar Colloquia series, Elsevier, New York, USA, 12, 127-135, 2002.

Chaston, C., Bonnell, J., Clausen, L., and Angelopoulos, V.: Energy transport by kinetic-scale electromagnetic waves in fast plasma sheet flows, J. Geophys. Res.-Space, 117, https://doi.org/10.1029/2012JA017863, 2012.

Dmitriev, A. V. and Suvorova, A. V.: Traveling magnetopause distortion related to a large-scale magnetosheath plasma jet: THEMIS and ground-based observations, J. Geophys. Res., 117, A08217, https://doi.org/10.1029/2011JA016861, 2012.

Dmitriev, A. V. and Suvorova, A. V.: Large-scale jets in the magnetosheath and plasma penetration across the magnetopause: THEMIS observations, J. Geophys. Res., 120, 4423-4437, https://doi.org/10.1002/2014JA020953, 2015.

Eastwood, J. P., Balogh, A., Lucek, E. A., Mazelle, C., and Dandouras, I.: On the existence of Alfvén waves in the terrestrial foreshock, Ann. Geophys., 21, 1457-1465, https://doi.org/10.5194/angeo-21-1457-2003, 2003.

Eastwood, J. P., Balogh, A., Lucek, E. A., Mazelle, C., and Dandouras, I.: Quasi-monochromatic ULF foreshock waves as observed by the four-spacecraft Cluster mission: 1. Statistical properties, J. Geophys. Res.-Space, 110, a11219, https://doi.org/10.1029/2004JA010617, 2005.

Ergun, R., Goodrich, K., Stawarz, J., Andersson, L., and Angelopoulos, V.: Large-amplitude electric fields associated with bursty bulk flow braking in the Earth's plasma sheet, J. Geophys. Res.-Space, 120, 1832-1844, https://doi.org/10.1002/2014JA020165, 2015.

Ergun, R., Tucker, S., Westfall, J., Goodrich, K., Malaspina, D., Summers, D., Wallace, J., Karlsson, M., Mack, J., Brennan, N., Pyke, B., Withnell, P., Torbert, R., Macri, J., Rau, D., Dors, I., Needell, J., Lindqvist, P.-A., Olsson, G., and Cully, C. M.: The axial double probe and fields signal processing for the MMS mission, Space Sci. Rev., 199, 167-188, 2016.

Fuselier, S., Klumpar, D., and Shelley, E.: On the origins of energetic ions in the Earth's dayside magnetosheath, J. Geophys. Res.-Space, 96, 47-56, 1991.

Fuselier, S., Lewis, W., Schiff, C., Ergun, R., Burch, J., Petrinec, S., and Trattner, K.: Magnetospheric multiscale science mission profile and operations, Space Sci. Rev., 199, 77-103, 2016.

Fuselier, S. A.: Suprathermal ions upstream and downstream from the Earth's bow shock, Solar wind sources of magnetospheric ultra-low-frequency waves, edited by: Engebretson, M. J., Takahashi, K., and Scholer, M., Geophysical Monograph, 81, 107119, American Geophysical, Union, Washington, USA, 1994.

Gary, S. P. and Eastman, T. E.: The lower hybrid drift instability at the magnetopause, J. Geophys. Res.-Space, 84, 7378-7381, https://doi.org/10.1029/JA084iA12p07378, 1979.

Gunell, H., Nilsson, H., Stenberg, G., Hamrin, M., Karlsson, T., Maggiolo, R., André, M., Lundin, R., and Dandouras, I.: Plasma penetration of the dayside magnetopause, Phys. Plasmas, 19, 072906, https://doi.org/10.1063/1.4739446, 2012.

Gunell, H., Stenberg Wieser, G., Mella, M., Maggiolo, R., Nilsson, H., Darrouzet, F., Hamrin, M., Karlsson, T., Brenning, N., De Keyser, J., André, M., and Dandouras, I.: Waves in high-speed plasmoids in the magnetosheath and at the magnetopause, Ann. Geophys., 32, 991-1009, https://doi.org/10.5194/angeo-32-9912014, 2014.

Gutynska, O., Sibeck, D. G., and Omidi, N.: Magnetosheath plasma structures and their relation to foreshock processes, J. Geophys. Res.-Space, 120, 7687-7697, https://doi.org/10.1002/2014JA020880, 2015.

Han, D.-S., Hietala, H., Chen, X.-C., Nishimura, Y., Lyons, L. R., Liu, J.-J., Hu, H.-Q., and Yang, H.-G.: Observational properties of dayside throat aurora and implications on the possible generation mechanisms, J. Geophys. Res.-Space, 122, 1853-1870, https://doi.org/10.1002/2016JA023394, 2017.

Hietala, H. and Plaschke, F.: On the generation of magnetosheath high-speed jets by bow shock ripples, J. Geophys. Res., 118, 7237-7245, https://doi.org/10.1002/2013JA019172, 2013.

Hietala, H., Laitinen, T. V., Andréeová, K., Vainio, R., Vaivads, A., Palmroth, M., Pulkkinen, T. I., Koskinen, H. E. J., Lucek, E. A., and Rème, H.: Supermagnetosonic Jets behind a Collisionless Quasiparallel Shock, Phys. Rev. Lett., 103, 245001, https://doi.org/10.1103/PhysRevLett.103.245001, 2009. 
Hietala, H., Partamies, N., Laitinen, T. V., Clausen, L. B. N., Facskó, G., Vaivads, A., Koskinen, H. E. J., Dandouras, I., Rème, H., and Lucek, E. A.: Supermagnetosonic subsolar magnetosheath jets and their effects: from the solar wind to the ionospheric convection, Ann. Geophys., 30, 33-48, https://doi.org/10.5194/angeo30-33-2012, 2012.

Hietala, H., Phan, T. D., Angelopoulos, V., Oieroset, M., Archer, M. O., Karlsson, T., and Plaschke, F.: In situ observations of a magnetosheath high-speed jet triggering magnetopause reconnection, Geophys. Res. Lett., 45, 1732-1740, https://doi.org/10.1002/2017GL076525, 2018.

Hoppe, M. M. and Russell, C. T.: Plasma rest frame frequencies and polarizations of the low-frequency upstream waves ISEE 1 and 2 observations, J. Geophys. Res., 88, 2021-2027, https://doi.org/10.1029/JA088iA03p02021, 1983.

Huba, J. D., Gladd, N. T., and Papadopoulos, K.: Lowerhybrid-drift wave turbulence in the distant magnetotail, J. Geophys. Res.-Space, 83, 5217-5226, https://doi.org/10.1029/JA083iA11p05217, 1978.

Karimabadi, H., Roytershteyn, V., Vu, H., Omelchenko, Y., Scudder, J., Daughton, W., Dimmock, A., Nykyri, K., Wan, M., Sibeck, D., et al.: The link between shocks, turbulence, and magnetic reconnection in collisionless plasmas, Phys. of Plasmas, 21, 062308, https://doi.org/10.1063/1.4882875, 2014.

Karlsson, T., Brenning, N., Nilsson, H., Trotignon, J.-G., Vallières, X., and Facsko, G.: Localized density enhancements in the magnetosheath: Three-dimensional morphology and possible importance for impulsive penetration, J. Geophys. Res., 117, A03227, https://doi.org/10.1029/2011JA017059, 2012.

Karlsson, T., Hamrin, M., Nilsson, H., Kullen, A., and Pitkänen, T.: Magnetic forces associated with bursty bulk flows in Earth's magnetotail, Geophy. Res. Lett., 42, 3122-3128, https://doi.org/10.1002/2015GL063999, 2015a.

Karlsson, T., Kullen, A., Liljeblad, E., Brenning, N., Nilsson, H., Gunell, H., and Hamrin, M.: On the origin of magnetosheath plasmoids and their relation to magnetosheath jets, J. Geophys. Res., 120, 7390-7403, https://doi.org/10.1002/2015JA021487, 2015 b.

Karlsson, T., Eriksson, A. I., Odelstad, E., André, M., Dickeli, G., Kullen, A., Lindqvist, P.-A., Nilsson, H., and Richter, I.: Rosetta measurements of lower hybrid frequency range electric field oscillations in the plasma environment of comet 67P, Geophys. Res. Lett., 44, 1641-1651, 2017.

Katırcıoglu, F. T., Kaymaz, Z., Sibeck, D. G., and Dandouras, I.: Magnetosheath cavities: case studies using Cluster observations, Ann. Geophys., 27, 3765-3780, https://doi.org/10.5194/angeo27-3765-2009, 2009.

LASP: MMS data, available at: https://lasp.colorado.edu/mms/sdc/ public/, last access: 10 April 2018.

Le Contel, O., Leroy, P., Roux, A., Coillot, C., Alison, D., Bouabdellah, A., Mirioni, L., Meslier, L., Galic, A., Vassal, M., et al.: The search-coil magnetometer for MMS, Space Sci. Rev., 199, 257-282, 2016.

Lemaire, J.: Plasmoid motion across a tangential discontinuity (with application to the magnetopause), J. Plasma Phys., 33, 425-436, 1985.

Lin, Y., Lee, L. C., and Yan, M.: Generation of dynamic pressure pulses downstream of the bow shock by variations in the inter- planetary magnetic field orientation, J. Geophys. Res., 101, 479493, https://doi.org/10.1029/95JA02985, 1996a.

Lin, Y., Swift, D. W., and Lee, L. C.: Simulation of pressure pulses in the bow shock and magnetosheath driven by variations in interplanetary magnetic field direction, J. Geophys. Res., 101, 2725127269, https://doi.org/10.1029/96JA02733, 1996b.

Lindqvist, P.-A., Olsson, G., Torbert, R., King, B., Granoff, M., Rau, D., Needell, G., Turco, S., Dors, I., Beckman, P., Macri, J., Frost, C., Salwen, J., Eriksson, A., Åhlén, L., Khotyaintsev, Y. V., Porter, J., Lappalainen, K., Ergun, R. E., Wermeer, W., and Tucker, S.: The spin-plane double probe electric field instrument for MMS, Space Sci. Rev., 199, 137-165, 2016.

Lucek, E., Constantinescu, D., Goldstein, M., Pickett, J., Pinçon, J., Sahraoui, F., Treumann, R., and Walker, S.: The magnetosheath, in: Outer Magnetospheric Boundaries: Cluster Results, Springer, Berlin, Germany, 95-152, 2005.

Lucek, E. A., Horbury, T. S., Dunlop, M. W., Cargill, P. J., Schwartz, S. J., Balogh, A., Brown, P., Carr, C., Fornacon, K.H., and Georgescu, E.: Cluster magnetic field observations at a quasi-parallel bow shock, Ann. Geophys., 20, 1699-1710, https://doi.org/10.5194/angeo-20-1699-2002, 2002.

Mailyan, B., Munteanu, C., and Haaland, S.: What is the best method to calculate the solar wind propagation delay?, Ann. Geophys., 26, 2383-2394, https://doi.org/10.5194/angeo26-2383-2008, 2008.

Němeček, Z., Šafránková, J., Přech, L., Sibeck, D. G., Kokubun, S., and Mukai, T.: Transient flux enhancements in the magnetosheath, Geophys. Res. Lett., 25, 1273-1276, https://doi.org/10.1029/98GL50873, 1998.

Norgren, C., Vaivads, A., Khotyaintsev, Y. V., and André, M.: Lower Hybrid Drift Waves: Space Observations, Phys. Rev. Lett., 109, 055001, https://doi.org/10.1103/PhysRevLett.109.055001, 2012.

Pickett, J. S., Menietti, J. D., Gurnett, D. A., Tsurutani, B., Kintner, P. M., Klatt, E., and Balogh, A.: Solitary potential structures observed in the magnetosheath by the Cluster spacecraft, Nonlin. Processes Geophys., 10, 3-11, https://doi.org/10.5194/npg-10-32003, 2003.

Plaschke, F., Glassmeier, K.-H., Sibeck, D. G., Auster, H. U., Constantinescu, O. D., Angelopoulos, V., and Magnes, W.: Magnetopause surface oscillation frequencies at different solar wind conditions, Ann. Geophys., 27, 4521-4532, https://doi.org/10.5194/angeo-27-4521-2009, 2009.

Plaschke, F., Hietala, H., and Angelopoulos, V.: Anti-sunward highspeed jets in the subsolar magnetosheath, Ann. Geophys., 31, 1877-1889, https://doi.org/10.5194/angeo-31-1877-2013, 2013.

Plaschke, F., Hietala, H., Angelopoulos, V., and Nakamura, R.: Geoeffective jets impacting the magnetopause are very common, J. Geophys. Res., 121, 3240-3253, https://doi.org/10.1002/2016JA022534, 2016.

Plaschke, F., Karlsson, T., Hietala, H., Archer, M., Vörös, Z., Nakamura, R., Magnes, W., Baumjohann, W., Torbert, R. B., Russell, C. T., and Giles, B. L.: Magnetosheath High-Speed Jets: Internal Structure and Interaction With Ambient Plasma, J. Geophys. Res.-Space, 122, 10157-10175, https://doi.org/10.1002/2017JA024471, 2017.

Pollock, C., Moore, T., Jacques, A., et al.: Fast plasma investigation for magnetospheric multiscale, Space Sci. Rev., 199, 331-406, 2016. 
Roth, M.: On impulsive penetration of solar wind plasmoids into the geomagnetic field, Plane. Space Sci., 40, 193-201, 1992.

Russell, C., Anderson, B., Baumjohann, W., Bromund, K., Dearborn, D., Fischer, D., Le, G., Leinweber, H., Leneman, D., Magnes, W., et al.: The magnetospheric multiscale magnetometers, Space Sci. Rev., 199, 189-256, 2016.

Savin, S., Amata, E., Zelenyi, L., Budaev, V., Consolini, G., Treumann, R., Lucek, E., Safrankova, J., Nemecek, Z., Khotyaintsev, Y., Andre, M., Buechner, J., Alleyne, H., Song, P., Blecki, J., Rauch, J. L., Romanov, S., Klimov, S., and Skalsky, A.: High energy jets in the Earth's magnetosheath: Implications for plasma dynamics and anomalous transport, J. Exp. Theor. Phys.+, 87, 593-599, https://doi.org/10.1134/S0021364008110015, 2008.

Savin, S., Amata, E., Zelenyi, L., Lutsenko, V., Safrankova, J., Nemecek, Z., Borodkova, N., Buechner, J., Daly, P. W., Kronberg, E. A., Blecki, J., Budaev, V., Kozak, L., Skalsky, A., and Lezhen, L.: Super fast plasma streams as drivers of transient and anomalous magnetospheric dynamics, Ann. Geophys., 30, 1-7, https://doi.org/10.5194/angeo-30-1-2012, 2012.

Schwartz, S. J. and Burgess, D.: Quasi-parallel shocks - A patchwork of three-dimensional structures, Geophys. Res. Lett., 18, 373-376, https://doi.org/10.1029/91GL00138, 1991.

Shue, J.-H., Chao, J.-K., Song, P., McFadden, J. P., Suvorova, A., Angelopoulos, V., Glassmeier, K. H., and Plaschke, F.: Anomalous magnetosheath flows and distorted subsolar magnetopause for radial interplanetary magnetic fields, Geophys. Res. Lett., 36, L18112, https://doi.org/10.1029/2009GL039842, 2009.
Stawarz, J., Ergun, R., and Goodrich, K.: Generation of highfrequency electric field activity by turbulence in the Earth's magnetotail, J. Geophys. Res.-Space, 120, 1845-1866, 2015.

THEMIS: THEMIS data and analysis software (SPEDAS), available at: http://themis.ssl.berkeley.edu, last access: 10 April 2018.

Torbert, R., Russell, C., Magnes, W., et al.: The FIELDS instrument suite on MMS: Scientific objectives, measurements, and data products, Space Sci. Rev., 199, 105-135, 2016.

Tsurutani, B. T. and Rodriguez, P.: Upstream waves and particles: An overview of ISEE results, J. Geophys. Res.-Space, 86, 43174324, 1981.

Vogt, J., Paschmann, G., and Chanteur, G.: Reciprocal vectors, ISSI SR-008, 33-46, 2008.

Voitcu, G. and Echim, M.: Tangential deflection and formation of counterstreaming flows at the impact of a plasma jet on a tangential discontinuity, Geophys. Res. Lett., 44, 5920-5927, https://doi.org/10.1002/2017GL073763, 2017.

Wilson, L. B.: Low Frequency Waves at and Upstream of Collisionless Shocks, John Wiley \& Sons Inc., Hoboken, NJ, USA, 269-291, https://doi.org/10.1002/9781119055006.ch16, 2016. 